\title{
Der Horber Vertrag vom 10. Juni 1498
}

\author{
Eine Untersuchung zu seiner Genese
}

Von Axel Metz

\section{Einleitung}

Die Absetzung Herzog Eberhards II. von Württemberg nach nur zweijähriger Herrschaft im Frühjahr 1498, die einem Zusammenwirken seiner Räte, der württembergischen Landstände und König Maximilians I. geschuldet war ${ }^{1}$, hinterließ bereits bei den Zeitgenossen einen tiefen Eindruck. Die Erinnerung an diese Ereignisse wurde denn auch schon nach wenigen Jahren von verschiedenen politischen Akteuren für die eigene Argumentation genutzt. So dienten die Vorgänge um die Absetzung Eberhards 1514 einem gewissen Schurlin in Leonberg während der Aufstandsbewegung des Armen Konrad als Beleg dafür, dass die Interessen des „gemeinen Mannes“ bei Herrschaftsstreitigkeiten immer wieder übergangen würden $^{2}$. Noch stärker instrumentalisierte Eberhards Neffe und Nachfolger, Herzog Ulrich, die Ereignisse von 1498, indem er sein Vorgehen gegen Konrad und Sebastian Breuning, Konrad Vaut sowie andere Vertreter der württembergischen „Ehrbarkeit" ab 1516 unter anderem damit rechtfertigte, dass diese Personen anfenger und die fürtrefflichsten ursächer seyen bemelts unsers vettern seliger verjagens ${ }^{3}$.

1 Vgl. hierzu: Axel Metz, Der Stände oberster Herr. Königtum und Landstände im süddeutschen Raum zur Zeit Maximlians I. (Veröffentlichungen der Kommission für geschichtliche Landeskunde in Baden-Württemberg, Reihe B, Bd. 174), Stuttgart 2009, S. 109-128. Der Begriff „König“ ist in der vorliegenden Untersuchung außer für das Reichsoberhaupt selbst auch als Chiffre zu verstehen für die die königliche Politik jeweils bestimmenden Gruppierungen am Hof, ohne dass diese stets namhaft gemacht werden können.

2 Vgl. dazu den Bericht des Leonberger Vogts vom 25. Juni 1514 über die Vorgänge in der Stadt (HStA Stuttgart A 45 Bü 3), in dem er die Äußerungen Schurlins wie folgt wiedergibt: Man hat uns vormalls unnd insonnder da man hertzog Eberharten vertriben hat, auch guote wort geben unnd placebo gemacht, bis man unns in das spil gebracht hat. Unnd man gyt uns yezo aber guote wort, aber wir werden unns nit mer daran kern.

${ }^{3}$ Ausschreiben Ulrichs vom 8. Januar 1519: Christian Friedrich SatTler, Geschichte des Herzogthums Würtenberg unter der Regierung der Herzogen, Teil 1, Tübingen 1769, Beyl. 103. Zum geringen Wahrheitsgehalt dieser Aussage vgl. Metz (wie Anm. 1) S. 168-174. 
Auch in der württembergischen Historiographie fand der Herrschaftsübergang von Eberhard auf Ulrich stets große Aufmerksamkeit ${ }^{4}$. Daher verwundert es nicht, dass das Dokument, das Eberhards de facto-Absetzung besiegelte, der Horber Vertrag vom 10. Juni 1498, wiederholt ediert wurde ${ }^{5}$.

Die Beschäftigung mit dem Vertrag erfolgte indes bisher lediglich auf Grundlage der ausgefertigten Urkunde Maximilians ${ }^{6}$, die sich heute in zwei Exemplaren im Hauptstaatsarchiv Stuttgart befindet ${ }^{7}$. Der Forschung entging dabei indes, dass zwei Entwürfe des Vertrags existieren, die im Tiroler Landesarchiv (TLA) Innsbruck aufbewahrt werden und unterschiedliche Vorstadien des ausgefertigten Textes repräsentieren ${ }^{8}$. Anhand dieser lässt sich nicht nur die Genese des Vertragstextes gut nachvollziehen, vielmehr ermöglicht der Textvergleich auch interessante und wichtige Rückschlüsse auf den vorausgegangenen Verhandlungsprozess. Die

${ }^{4}$ Vgl. dazu etwa: Johann Ulrich STEInhofer, Ehre des Herzogtums Wirtemberg In seinen Durchlauchtigsten Regenten, Oder Neue Wirtembergische Chronik, Bd.3, Stuttgart 1752, S. 679-773; Sattler (wie Anm. 3) S. 23-38; Ludwig Friedrich Heyd, Ulrich, Herzog zu Württemberg: Ein Beitrag zur Geschichte Württembergs und des deutschen Reiches im Zeitalter der Reformation, Bd. 1, Tübingen 1841, S.12-37; Christoph Friedrich von STÄLIN, Wirtembergische Geschichte, Vierter Theil: Schwaben und Südfranken vornehmlich im 16. Jahrhundert. Zeit der wirtembergischen Herzoge Eberhard II., Ulrich, Christoph, Ludwig 1498-1593, Stuttgart 1873, S. 8-23.

${ }^{5}$ Die Regesta Imperii XIV: Ausgewählte Regesten des Kaiserreiches unter Maximilian I. 1493-1519, bearb. von Hermann Wiesflecker, Wien/Köln 1990 ff., Nr. 6256 (künftig: RI + Nr.), nennen vier Editionen des Vertrags; am einfachsten zugänglich ist er bei: SATTLER (wie Anm. 3) Beyl. 15; ein ausführliches Regest mit Abdruck umfangreicher Textpassagen bieten die Württembergische Landtagsakten I498-I5I5, hg. von Wilhelm OHR/Erich Kober (Württembergische Landtagsakten, Reihe I, Bd. 1), Stuttgart 1913, Nr. 24, S. 94-96. Darüber hinaus ist das handschriftliche Original als Digitalisat auf den Internet-Seiten des Landesarchivs Baden-Württemberg abrufbar: https://www2.landesarchiv-bw.de/ofs21/bild_zoom/ thumbnails.php ? bestand $=3703 \&$ basisid $=150911 \&$ syssuche $=\& \operatorname{logik}=($ Stand: 23.6 .2013$)$.

${ }^{6}$ Diese Aussage trifft auch zu auf die Darstellung von Joachim Lipp, 500 Jahre Horber Vertrag. König Maximilian beendete im österreichischen Horb eine württembergische Regierungskrise, in: Freudenstädter Heimatblätter 29,7 (Juli 1998) S. 4. Lipp arbeitet außerdem mit sehr kraftvollen Ausdrücken, die der historischen Situation nicht immer angemessen erscheinen, etwa wenn er die Herrschaft Eberhards II. als „wahres Schreckensregiment“ bezeichnet, oder davon spricht, dass Herzog Eberhard im Bart die nach seinem Tod eintretende Situation „angesichts seiner degenerierten Verwandtschaft wohl vorausgesehen“ habe.

7 HStA Stuttgart A 602 Nr. 461 und 461a.

8 TLA Innsbruck, Maximiliana IVa/42 S. 1-8 und Maximiliana XIV/1498 Bl.170r-173r. Der zuletzt genannte Text hat Eingang gefunden in die Kurzregesten des einschlägigen Regesta Imperii-Bandes zu Maximilian I. (Nr. 6242) sowie - gleichfalls als Regest - in die Edition der Reichstagsakten: Deutsche Reichstagsakten unter Maximilian I., Bd. 6: Reichstage von Lindau, Worms und Freiburg 1496-1498, bearb. von Heinz Gollwitzer (Deutsche Reichstagsakten, Mittlere Reihe, Bd.6), Göttingen 1979, Nr. 145; als genetische Vorstufe des Horber Vertrags wird der Text darin indes jeweils nicht benannt. Der Verfasser des vorliegenden Beitrags wies auf die beiden Konzepte des Horber Vertrags in einer früheren Arbeit hin, freilich ohne dass ihm in diesem Rahmen eine intensivere Beschäftigung damit möglich gewesen wäre. Vgl. dazu: Metz (wie Anm. 1) S. 124. 
zeitliche Einordnung ist dabei recht genau möglich, da der zweite Entwurf (TLA Innsbruck, Maximiliana XIV/1498, Bl.170r-173r) auf den 6. Juni 1498 datiert ist. Die dort durchgestrichenen Passagen verweisen inhaltlich auf den ersten Entwurf (TLA Innsbruck, Maximiliana IVa/42, S.1-8), der somit vorher entstanden sein muss. Da Ulrich bereits in diesem zeitlich ersten Text als Herzog angesprochen wird, ist das Datum der Verleihung des Herzogtums an ihn durch Maximilian am 28. Mai $1498^{9}$ als terminus post quem für die Entstehung des ersten Entwurfs anzusehen. Die zu vermutende vorausgegangene Intensität der Verhandlungen lässt dabei eher auf einen Termin schließen, der näher am 6. Juni als am 28. Mai 1498 liegt.

Im Folgenden soll nun kurz die Vorgeschichte des Horber Vertrages dargestellt werden. Daran schließt sich eine Beschreibung der drei Archivalien an, bevor eine Betrachtung der inhaltlichen Unterschiede und der daraus möglichen Rückschlüsse auf den Verhandlungsprozess erfolgen wird. Den Abschluss der Untersuchung bilden ein kurzes Resümee sowie ein Anhang, der eine Synopse der drei Texte bietet, um so einen besseren Vergleich zu ermöglichen, aber auch um die weitere Beschäftigung mit ihnen anzuregen.

\section{Vorgeschichte}

Der folgende kurze Überblick über den historischen Hintergrund des Horber Vertrags soll das Verständnis der sich anschließenden Ausführungen erleichtern. Eine detaillierte Darstellung kann mit Blick auf die vorhandene einschlägige Literatur indes unterbleiben ${ }^{10}$.

1482 gelang es Eberhard im Bart, Württemberg nach vierzigjähriger Teilung durch vertragliche Vereinbarung mit seinem Vetter Eberhard dem Jüngeren (d.J.), dem späteren Herzog Eberhard II., wiederzuvereinigen ${ }^{11}$. Allerdings kam es über die Ausgestaltung der Vereinbarung rasch zu Irrungen und gewalttätigen Auseinandersetzungen zwischen den beiden Cousins, die erst 1492 im Esslinger Vertrag endgültig beigelegt werden konnten ${ }^{12}$. Dieser bestimmte, dass Eberhard im Bart ganz Württemberg allein regieren, Eberhard d. J. dagegen sein Alleinerbe sein sollte. Allerdings musste der jüngere Eberhard im Fall der Herrschaftsübernahme

9 HStA Stuttgart A 602 Nr. 718; Edition: Satt ler (wie Anm. 3) Beyl. 14.

10 Vgl. dazu vor allem Walter Grube, Der Stuttgarter Landtag 1457-1957. Von den Landständen zum demokratischen Parlament, Stuttgart 1957, S.43-62; Dieter MerTens, Art. Württemberg, in: Handbuch der baden-württembergischen Geschichte, Bd. 2: Die Territorien im Alten Reich, hg. von Meinrad Schанв u.a., Stuttgart 1995, S. 1-163, hier S. 55-68; dort auch Hinweise zur weiterführenden Literatur.

11 Münsinger Vertrag vom 14.12.1482: HStA Stuttgart A 602 Nr.321; ediert bei: August Ludwig Reyscher (Hg.), Vollständige, historisch und kritisch bearbeitete Sammlung der württembergischen Gesetze, Bd. 1, Stuttgart/Tübingen 1828, Nr. 7, S. 489-495.

12 Urkunde vom 2.9.1492: HStA Stuttgart A 602 Nr.360; Edition: Reyscher (wie Anm. 11) Nr. 11, S. 513-520. 
ein ständisches Regiment an seiner Seite akzeptieren, das in alltäglichen Dingen selbständig entscheiden durfte und auch zur Verhandlung wichtigster Gegenstände gemeinsam mit Eberhard bzw. - wenn er eine Beteiligung an den Beratungen ablehnen würde - sogar allein befugt war. Diese hausvertraglichen Regelungen wurden nicht nur von Kaiser Friedrich III. alsbald konfirmiert ${ }^{13}$, sondern auch anlässlich der Erhebung Württembergs zum Herzogtum auf dem Wormser Reichstag am 21. Juli 1495 von König Maximilian I. noch einmal ausdrücklich bestätigt ${ }^{14}$. Als indes Eberhard im Bart Ende Februar 1496 starb, bemühte sich sein Vetter in Gesprächen mit Maximilian, die Regelungen des Esslinger Vertrags auszuhebeln. Zwar hatte er damit insoweit Erfolg, als auf die Einsetzung des Regiments verzichtet wurde. Allerdings musste er im Gegenzug mehrere königliche Räte in seiner engsten Umgebung akzeptieren. Außerdem gelang es dem Habsburger, sich andere Räte Eberhards als Diener zu verpflichten ${ }^{15}$. Der neue Herzog war somit von Personen umgeben, die die königlichen Belange bei ihren Handlungen im Blick behielten. Auf diesem Wege vermochte es Maximilian zunächst, Eberhards Politik zu kontrollieren und ihn von Maßnahmen abzuhalten, die gegen die königlichen Interessen gerichtet waren.

Der württembergische Herzog war freilich nicht bereit, sich mit dieser Situation auf Dauer abzufinden. So ergriff er Anfang 1498 Maßnahmen gegen die enge Verbindung seiner Räte zum König. Außerdem übte er mit Hilfe von Vertrauten, insbesondere des Augustinermönchs Konrad Holzinger ${ }^{16}$, Druck auf die städtischen Eliten des Landes, die „Ehrbarkeit“, aus, um im Innern die herzogliche Position zu stärken. Auf diese Weise rief er freilich mehrere Gegner gleichzeitig gegen sich auf den Plan, deren gemeinsames Handeln schließlich seine Verdrängung von der Regierung zur Folge hatte. Den Auftakt hierzu bildete ein Landtag in Stuttgart ab Ende März 1498, den der württembergische Kanzler Gregor Lamparter und der Landhofmeister Wolfgang von Fürstenberg, der zugleich ein Rat Maximilians mit engsten Beziehungen an den Königshof war ${ }^{17}$, unter Umgehung Eberhards, aber mit Wissen des Habsburgers ausgeschrieben hatten. Auf diesem wurden zunächst

${ }_{13}$ HStA Stuttgart A 602 Nr. 362 (8.10.1492).

${ }^{14}$ HStA Stuttgart A 602 Nr. 711; Edition: Deutsche Reichstagsakten unter Maximilian I., Bd. 5: Reichstag von Worms 1495, bearb. von Heinz Angermeier (Deutsche Reichstagsakten, Mittlere Reihe, Bd. 5), Göttingen 1981, Nr. 1168, S. 914-919.

${ }_{15}$ Vgl. hierzu und zu den im Folgenden berichteten Verhandlungen des Stuttgarter Landtags: Metz (wie Anm. 1) S. 116-128.

$16 \mathrm{Zu}$ seiner Person: Dieter Stievermann, Der Augustinermönch Dr. Conrad Holtzinger - Kaplan, Rat und Kanzler des Grafen bzw. Herzogs Eberhard d. J. von Württemberg am Ende des 15. Jahrhunderts, in: Mittel und Wege früher Verfassungspolitik. Kleine Schriften 1, hg. von Josef Engel (Spätmittelalter und Frühe Neuzeit. Tübinger Beiträge zur Geschichtsforschung, Bd. 9), Stuttgart 1979, S. 356-405.

17 Sein Bruder Heinrich hatte die Stellung des königlichen Hofmarschalls inne. Vgl. zur Königsnähe des Bruderpaars auch: Sigmund Riezler, Art. Fürstenberg, Graf Heinrich VII. (geb. 1464) und Graf Wolfgang (geb. 3. April 1465), in: ADB, Bd. 8, Leipzig 1878, S. 223-226. 
die Durchführung der im Esslinger Vertrag vorgesehenen Ordnung und weitere, die Herrschaft Eberhards beschränkende Regelungen gefordert. Als der Herzog sich - wie aufgrund der Vorgeschichte zu erwarten - den Forderungen nicht beugte, sagten ihm seine Räte und andere Verpflichtete den Dienst auf, womit Eberhard, der überdies zu dieser Zeit das württembergische Territorium verließ, seiner Herrschaftsmöglichkeiten faktisch vollständig beraubt war ${ }^{18}$. Jeder dieser gegen den zweiten württembergischen Herzog gerichteten Schritte geschah in Abstimmung mit dem König. Dies gilt auch für den Vorschlag, die Herrschaftskrise durch Übertragung der Regierung auf Eberhards Neffen Ulrich zu lösen, den die den Landtag dominierenden Kräfte alsbald ins Spiel brachten und der mit dem Habsburger zumindest abgesprochen, wenn nicht gar von diesem initiiert war ${ }^{19}$.

Nach außen hin freilich bemühte sich Maximilian, das Bild eines über den Parteien stehenden, unabhängigen Vermittlers aufrechtzuerhalten. So verhandelte er noch Anfang Mai mit beiden Seiten in Ulm, wenngleich eine Entscheidung zuungunsten Eberhards schon gefallen war ${ }^{20}$. Dies wurde dann öffentlich sichtbar, als Maximilian sich auf seinem Weg zum Freiburger Reichstag Ende Mai nach Württemberg begab ${ }^{21}$, um dort aber nicht den nominell noch regierenden Eberhard, sondern Abgesandte des zwischenzeitlich vom Stuttgarter Landtag ernannten Regiments zu treffen. So war es nur konsequent, dass Maximilian bereits am 28. Mai 1498 den noch unmündigen Ulrich mit dem Herzogtum belehnte, das freilich bis zu seiner Volljährigkeit von Wolfgang von Fürstenberg, also einem Rat Maximilians, vermannt werden sollte ${ }^{22}$.

Damit war über die künftige Regierung des Landes faktisch bereits entschieden. Aus Sicht des Königs galt es nur noch, Eberhard zum „freiwilligen“ Regierungsverzicht zu bewegen. Doch auch diesbezüglich hatte man am Hof Maximilians schon klare Vorstellungen. Danach sollte Eberhard sich mit dem Richtung Freiburg weiterziehenden König im Hauptort der habsburgischen Grafschaft Hohenberg, der Stadt Rottenburg, treffen und dort seinen Verzicht auf die Regierung erklären ${ }^{23}$. Der Weg von Urach nach Rottenburg führte Maximilian und seinen Hof

18 HStA Stuttgart A 602 Nr. 451; Württembergische Landtagsakten (wie Anm. 5) Nr.5, S. 23-25; Mitteilung des Stuttgarter Landtags an die zu Freiburg versammelten Reichsstände; RI 8542.

19 Vgl. zu dieser Einschätzung: Metz (wie Anm. 1) S. 122.

20 So berichtete Hans Ungelter bereits damals an seine Heimatstadt Esslingen, dass Ulrich zum regierenden Herrn in Württemberg erhoben werden solle. Vgl. dazu: HStA Stuttgart H 53 Bü 84 Bl. 35r-37v; Deutsche Reichstagsakten, Bd. 6 (wie Anm. 8) Nr. 134, S. 585 f.

${ }^{21}$ Am 23. Mai 1498 traf er von Ulm über Ehingen kommend in Urach ein. Vgl. dazu: RI 6211-6214.

22 Belehnungsurkunde: HStA Stuttgart A 602 Nr.718; abgedruckt bei: SATtLer (wie Anm. 3) Beyl. 14. Revers der Urkunde durch Ulrich und sein Regiment vom gleichen Tag: HStA Stuttgart G 41 U 2.

${ }^{23}$ Schreiben Heinrichs von Fürstenberg an Hans von Werdenberg vom 28. Mai 1498: HStA Stuttgart A 602 Nr. 457; Württembergische Landtagsakten (wie Anm. 5) Nr. 18, S. 80 f. 
über die Reichsstadt Reutlingen und die württembergischen Klöster Einsiedel und Bebenhausen ${ }^{24}$. In dieser Zeit müssen Verhandlungen zumindest über das erste der beiden Konzepte gepflogen und dieses niedergeschrieben worden sein; dabei zeichnete sich schon hier ab, dass die entscheidende Urkunde für die Verdrängung Eberhards von der Herrschaft in die Gestalt eines königlichen Schiedsspruchs gekleidet werden sollte ${ }^{25}$, wodurch Maximilian den Anschein eines neutralen Vermittlers zumindest ein Stück weit aufrecht erhalten konnte. Möglicherweise wurden damals auch schon Gespräche über den Text des zweiten Konzepts geführt, das auf den 6. Juni datiert wurde und Rottenburg als Ausstellungsort nennt.

Am gleichen Tag erklärte Herzog Eberhard gegenüber dem König denn auch seine Bereitschaft, zugunsten seines lieben vetter graf Ulrichen von Wirtemberg von der Regierung aber nicht als verwürckt zurückzutreten ${ }^{26}$. Insofern schien es so, als könne der Prozess der Absetzung bzw. des Rücktritts Eberhards sehr rasch zu einem Ende gelangen. Dies war jedoch nicht der Fall. Vielmehr hatten beide Parteien, Eberhard wie auch das württembergische Regiment, noch Verhandlungsbedarf, wie die entsprechenden Änderungen der dann ausgefertigten Urkunde des Horber Vertrags - gegenüber dem Entwurf vom 6. Juni 1498 zeigen.

Insbesondere in der Zeit zwischen dem 6. und 9. Juni dürfte noch sehr intensiv um den Rücktritt des Herzogs und die entsprechenden Gegenleistungen gerungen worden sein. Ein Zeugnis dafür ist auch der mit dem Ausstellungsort Rottenburg (allerdings ohne ein Ausstellungsdatum) versehene Entwurf einer Urkunde Maximilians, in dem Herzog Ulrich bzw. - aufgrund seiner Minderjährigkeit - an seiner Statt Graf Wolfgang von Fürstenberg das Herzogtum unter Hinweis auf eine Vereinbarung zwischen Ulrich und Eberhard übergeben wird, und die Untertanen an Ulrich als neuen Herrn gewiesen werden ${ }^{27}$. Offensichtlich sollte mit dieser Urkunde dem Widerspruch begegnet werden, dass Ulrich schon am 28. Mai 1498 mit Württemberg belehnt worden war und damit zu einem Zeitpunkt, zu dem Eberhard der Regierung noch nicht entsetzt war bzw. auf diese verzichtet hatte. Freilich

24 Vgl. Heyd (wie Anm. 4) S.28, Anm. 43; Stälin (wie Anm. 4) S. 19.

${ }^{25}$ Zum Charakter des Horber Vertrags als königlicher Schiedsspruch vgl. auch: Jürgen Sydow, Zum Problem kaiserlicher Schiedsverfahren unter Maximilian I. Der Tübinger Vertrag von 1514 (Kleine Arbeitsreihe des Instituts für Europäische und Vergleichende Rechtsgeschichte an der Rechts- und Staatswissenschaftlichen Fakultät der Universität Graz, Heft 5), Graz 1973, S. 17.

${ }^{26}$ HStA Stuttgart A 602 Nr. 458. Mit der Bezeichnung des schon am 28. Mai 1498 von Maximilian mit dem Herzogtum belehnten Ulrich (vgl. Anm. 22) als Grafen wie auch mit seinem Insistieren darauf, dass er sein Fürstentum nicht verwirkt habe, wollte Eberhard offenkundig sein Gesicht wahren und seine Absetzung als freiwilligen Akt des Herrschaftsverzichts kennzeichnen. Wie indes die zu Eingang dieses Beitrags angeführten Charakterisierungen der Ereignisse durch Schurlin von Leonberg und Herzog Ulrich als Vertreibung bzw. Verjagung zeigen, war diesem Bemühen Eberhards um Gesichtswahrung kein Erfolg beschieden.

27 HStA Stuttgart A 602 Nr.459; in weiten Teilen abgedruckt bei: Württembergische Landtagsakten (wie Anm. 5) Nr. 22, S. 87-90. 
wurde diese Urkunde dann nicht ausgefertigt, vermutlich deshalb, weil man diejenige vom 28. Mai 1498 nicht entwerten wollte ${ }^{28}$. Immerhin unterstreicht dies aber, wie intensiv die Verhandlungen in Rottenburg waren ${ }^{29}$.

Ferner wurde dort gleichzeitig noch zwischen dem König und den Vertretern Ulrichs und seines Regiments um deren Gegenleistungen für Maximilians Mitwirken an der Vertreibung Eberhards verhandelt, die am 9. Juni schließlich schriftlich fixiert wurden ${ }^{30}$. Es ist davon auszugehen, dass sich Maximilian diese Zusagen zunächst bestätigen lassen wollte, ehe er seine endgültige Entscheidung im Streit zwischen Eberhard einerseits und Ulrich sowie dem Regiment andererseits öffentlich bekanntgab. Im Vorfeld des 9. Juni spielten sich somit die Verhandlungen um den königlichen Spruch wie auch um die Gegenleistungen des neuen Regiments für Maximilian parallel ab. Während es indes gelang, die Urkunde über die Zugeständnisse an den Habsburger schon am 9. Juni in Rottenburg auszustellen, wurde der königliche Spruch, der die Vereinbarungen zwischen Eberhard und Ulrich samt seinem Regiment für den Regierungsverzicht des zweiten württembergischen Herzogs schriftlich fixierte ${ }^{31}$, erst einen Tag später im gleichfalls hohenbergischen (und damit habsburgischen) Horb ausgefertigt ${ }^{32}$, wohin der König auf seinem Weg zum Freiburger Reichstag weitergezogen war ${ }^{33}$.

${ }^{28}$ Die Herausgeber der Württembergischen Landtagsakten (wie Anm. 5) S. 87 f., Anm. 4, sehen in dem Text „eine Vorarbeit zum Horber Vertrag“ und datieren ihn auf den 9. Juni 1498. Es ist indes nicht nur davon auszugehen, dass sie damit die Entstehung des Texts zeitlich zu spät einordnen; vielmehr trifft auch der Terminus „Vorarbeit" den Charakter des Schriftstücks im Lichte der beiden in Innsbruck aufbewahrten Konzepte kaum richtig. Eher handelt es sich um den Entwurf einer Nebenurkunde zum Horber Vertrag, die den Herrschaftsübergang absichern sollte, dann jedoch mit Blick auf die Urkunde vom 28. Mai 1498 zumindest Ulrich und seinem Regiment, möglicherweise aber auch dem König als verzichtbar, wenn nicht gar als kontraproduktiv erschien.

${ }^{29}$ Einen weiteren Hinweis auf die Intensität dieser Verhandlungen liefert auch die an den König gerichtete Beschwerde Eberhards gegen den Horber Vertrag vom 16. Juni 1498 (HStA Stuttgart A 602 Nr. 464), in der er unter anderem von seiner schrifftlichen und muntlichen warlichen verantwurttung gegenüber Maximilian spricht und der Gegenseite vorwirft, im Rahmen der Vertragsentstehung mit unordnung [...] wider uns conspiriret und verhanndelt zu haben. - Dabei ist zu bedenken, dass die Verhandlungen mit den beiden württembergischen Parteien neben den anderen Regierungsgeschäften des Königs herliefen. So berichtete etwa der spanische Gesandte Gomez de Fuensalida, dass er in diesen Tagen eine fast fünfstündige Audienz bei Maximilian hatte: RI 6252.

30 HStA Stuttgart A 602 Nr. 458.

${ }^{31}$ HStA Stuttgart A 602 Nr. 461 und 461a.

32 Eberhard selbst erklärte seinen offiziellen Regierungsverzicht gegenüber Maximilian dann noch einen Tag später, am 11. Juni, gleichfalls in Horb; HStA Stuttgart A 602 Nr. 462.

33 Dass damit die württembergischen Händel aus der Sicht Maximilians, ungeachtet der bald darauf von Eberhard erhobenen Beschwerden über den Vertrag (vgl. dazu: HStA Stuttgart A 602 Nr. 464), beendet waren, zeigt der Umstand, dass er sich nun sehr rasch Freiburg näherte. Bereits am 14. Juni war er in Kenzingen angelangt, wo er einige Tage verweilte, um den königlichen Adventus in der Reichstagsstadt vorzubereiten. Vgl. dazu: Ulrich P. EckeR, „... sitzen untätig herum, verhandeln nichts, aber verzehren viel Geld“. Organisation und 


\section{Beschreibung der Archivalien}

Im Folgenden ist nun kurz auf die äußere Form der drei zu behandelnden Archivalien einzugehen, bevor die inhaltlichen Abweichungen zwischen den Texten im Fokus der Betrachtung stehen sollen. Bei dem ersten hier interessierenden Archivale, das sich im Tiroler Landesarchiv befindet und dort die Signatur Maximiliana IVa/42 trägt, handelt es sich um einen achtseitigen Text auf Papier mit in neuerer Zeit hinzugefügter Paginierung, der die typischen Merkmale eines Konzepts, wie breiten Korrekturrand, Streichungen und Ergänzungen sowie den Verzicht auf einige formularartige Urkundenbestandteile (z. B. die Intitulatio) aufweist. Der Text ist nur fragmentarisch erhalten und endet mitten in einem Satz. Allerdings fehlen im Wesentlichen vermutlich nur einige relativ starre Bestandteile am Urkundenende (Sanctio, Corroboratio, Eschatokoll), so dass mit dem Verlust lediglich einer Seite zu rechnen ist. Bereits der Lagerungsort weist darauf hin, dass der Text in der Kanzlei Maximilians entstanden sein dürfte und anschließend als Grundlage für die Abstimmung mit den beiden württembergischen Parteien genutzt wurde. Wie bereits erwähnt, handelt es sich um den zeitlich ersten noch erhaltenen Entwurf des Horber Vertrags, für dessen Entstehung der Zeitraum zwischen dem 28. Mai und dem 6. Juni 1498 anzunehmen ist. Diese Datierung ergibt sich aus den bereits am Ende des einleitenden Abschnitts erwähnten inneren Merkmalen. Umfangreichere Änderungen, und zwar Ergänzungen, weist der Entwurf nur auf den Seiten 3 und 6 auf, wobei diese Modifikationen vor allem Interessen Ulrichs und seines Regiments (zuungunsten Eberhards) berücksichtigen. Daher ist davon auszugehen, dass die Ergänzungen nach Gesprächen des Königs bzw. seines Umfelds mit den Vertretern des neuen württembergischen Regiments in den Text eingefügt wurden. Freilich finden sich nicht alle im zweiten Konzept wieder; dieser Umstand wie auch andere Änderungen des zweiten Konzepts im Vergleich zum ersten deuten darauf hin, dass Eberhard vor der Abfassung des zweiten Konzepts die Möglichkeit hatte, intensiver auf den Vertragstext einzuwirken.

Dieses zweite Konzept befindet sich gleichfalls im Tiroler Landesarchiv, und zwar in der Akte Maximiliana XIV/1498 auf Blatt 170-173, wobei die Foliierung modernen Ursprungs ist. Der Text ist gleichfalls auf Papier geschrieben, aber von einer anderen Hand als das erste Konzept und weist ebenfalls die typischen Konzeptmerkmale auf. $\mathrm{Zu}$ beachten ist, dass der fortlaufende Text auf Blatt 171r beginnt und bis Blatt 173r reicht. Der Text des Blattes 170 dagegen ist eine Ergänzung, deren Einfügungsort auf Blatt 172r eindeutig markiert ist ${ }^{34}$. Auch dieser Text

Ablauf des Freiburger Reichstags, in: Der Kaiser in seiner Stadt. Maximilian I. und der Reichstag zu Freiburg 1498, hg. von Hans SCHAdEK, Freiburg i. Br. 1998, S. 56-93, hier S. 82 f.

${ }^{34}$ Inwieweit die Herausgeber der Regesta Imperii und des einschlägigen Reichstagsaktenbandes (vgl. Anm. 8) dies berücksichtigt haben, ist unklar, da sie beide nur die Blätter 171 und 172f. nennen - und zwar in dieser etwas überraschenden Form statt 171-173. Die Reichstagsakten verweisen überdies auf Nr.458 der Württembergischen Regesten als ge- 
weist verhältnismäßig wenige Streichungen auf; allerdings sind mehrere Ergänzungen eingefügt, die bei Weitem längste findet sich auf einem eigenen Blatt, dem soeben genannten Blatt 170, wobei dort wiederum umfangreichere Streichungen und Änderungen vorgenommen wurden. Die zeitliche Stellung zwischen dem ersten Konzept und der Ausfertigung des Horber Vertrags vom 10. Juni ergibt sich daraus, dass der Text in seinen gestrichenen Passagen Parallelen zum ersten Entwurf aufweist, während die an deren Stelle tretenden bzw. ergänzten Passagen sich vor allem in der Ausfertigung finden. Dies bedeutet, dass der Text entstanden ist, um die von den Parteien am ersten Konzept gewünschten Änderungen aufzunehmen und zugleich als Grundlage für den weiteren Verhandlungsprozess zu dienen. In dessen Rahmen wurden verschiedene Änderungen gewünscht, die als Korrekturen und Nachträge in das zweite Konzept einflossen. Weitere Verhandlungen führten schließlich zur Klärung letzter noch offener Punkte, bevor die Ausfertigung durch die Kanzlei Maximilians veranlasst werden konnte.

Allerdings gibt es eine längere Passage, die sich zwar im ersten Konzept und in der Ausfertigung findet, jedoch nicht im zweiten Konzept: Hierbei handelte es sich um die Erzählung der Vorgeschichte der Urkundenausfertigung („Narratio“); mit dieser waren vermutlich sowohl Ulrich und sein Regiment als auch Eberhard einverstanden, weswegen man hier keine Notwendigkeit sah, sie im zweiten Konzept eigens aufzuführen. Denkbar wäre darüber hinaus, dass Maximilian bzw. seine Kanzlei die Formulierung der Narratio (weitgehend) für sich selbst beanspruchten und sie daher nicht in das als Diskussionsgrundlage gedachte zweite Konzept aufnahmen.

Von der schließlich ausgefertigten Urkunde sind zwei Exemplare erhalten, die sich heute im Bestand der Württembergischen Regesten des Hauptstaatsarchivs Stuttgart (A 602 Nr. 461 und 461a) befinden; ganz offensichtlich handelt es sich dabei zum einen um die Ausfertigung für Ulrich und sein Regiment sowie zum anderen um diejenige für Eberhard II., erhielten doch beide Parteien - wie der Urkundentext selbst belegt - jeweils ein Exemplar. Die beiden je achtseitigen, mit dem königlichen Majestätssiegel beglaubigten Pergamentlibelle fanden zunächst Eingang in das württembergische Hausarchiv, bevor sie bei der Etablierung der Württembergischen Regesten Ende des 19. Jahrhunderts in diese eingeordnet wurden ${ }^{35}$. Textlich sind beide Urkunden - abgesehen von versehentlichen Schreibvarianten

druckte Version dieses zweiten Entwurfs. Dabei handelt es sich jedoch um ein gänzlich anderes Dokument (vgl. Anm. 26).

${ }^{35}$ Vgl. zur Bildung des Bestands A 602 (Württembergische Regesten) des Hauptstaatsarchivs Stuttgart: Stephan Molitor, Projektmanagement avant la lettre. Gerhard Mehring (1864-1931) und die Württembergischen Regesten, in: Archivisches Arbeiten im Umbruch. Vorträge des Kolloquiums der staatlichen Archivverwaltung Baden-Württemberg am 26. und 27. November 2002 im Staatsarchiv Ludwigsburg aus Anlass der Verabschiedung von Herrn Professor Dr. Gerhard Taddey, hg. von Norbert Hoffman/Stephan Molitor, Stuttgart 2004, S. 35-43. 
und einer leicht divergierenden Raumaufteilung - gleich ${ }^{36}$. Wie bereits erwähnt, wurde die Urkunde außerdem wiederholt ediert und ist zwischenzeitlich als Digitalisat im Internet einsehbar ${ }^{37}$.

\section{Inhaltliche Untersuchung der Archivalien und daraus resultierende Rückschlüsse auf den Verhandlungsprozess}

Betrachtet man die drei Archivalien inhaltlich, so werden Parallelen wie auch Veränderungen sichtbar, die Rückschlüsse auf den Verhandlungsprozess zulassen, der schließlich zum ausgefertigten Vertragstext vom 10. Juni 1498 geführt hat. Das erste Element im Aufbau der Urkunde, die Intitulatio Maximilians, ist freilich in dieser Beziehung noch wenig aussagekräftig. Ihr Fehlen in den beiden Konzepten gegenüber der Ausfertigung erklärt sich leicht daraus, dass sie zu den formularartigen Urkundenbestandteilen gehörte, somit nicht Gegenstand von Verhandlungen sein musste und erst von der königlichen Kanzlei in die Urkunde vom 10. Juni aufgenommen wurde.

Erste Hinweise auf den Verhandlungsprozess bietet dagegen die Erzählung der Vorgeschichte des Vertrages (Narratio), die freilich im zweiten Konzept weitgehend fehlt. Auffällig ist, dass der Ursprungstext des ersten Konzepts sich inhaltlich weitgehend mit dem der ausgefertigten Urkunde deckt. Allerdings erfuhr das erste Konzept eine Ergänzung, die jedoch keinen Eingang in die Ausfertigung gefunden hat. Konkret handelt es sich um eine Strafandrohung gegen Eberhard, wonach der König auf Anrufen Ulrichs und seines Regiments entscheiden könne, dass Eberhard gegen den Vertrag verstoßen habe mit der Folge, dass dadurch seine Ansprüche auf die noch zu betrachtende Pension verwirkt seien. Dieser Punkt sollte also Ulrich und sein Regiment gegen Eberhards Handeln absichern; es ist daher sehr wahrscheinlich, dass er auf ihre Intervention zunächst in das erste Konzept hineingeschrieben, auf Eberhards Widerstand hiergegen bei der Abfassung der Ausfertigung nicht berücksichtigt wurde.

Es folgt in allen drei Texten der Entscheid Maximilians, wonach Eberhard auf das Herzogtum verzichten und es zu Handen Ulrichs stellen solle, den der König damit belehnt. Diese Passage weist in allen drei Fassungen große Ähnlichkeiten auf. Zwei Punkte erscheinen indes beachtenswert: So sprechen sowohl das erste

${ }^{36}$ Die Edition im Anhang richtet sich nach A 602 Nr. 461, da dieses Exemplar - anders als Nr. 461a - eine Vielzahl von Registraturvermerken aufweist und in der württembergischen Historiographie als das Exemplar des Horber Vertrages angesehen wurde. Es erscheint daher wahrscheinlich, dass es sich dabei um die Ausfertigung für Ulrich und das Regiment handelt, mit der intensiver gearbeitet wurde, während dasjenige für Eberhard eher ein Schattendasein führte. Neben den beiden Ausfertigungen findet sich in den Württembergischen Regesten noch eine weitere Version dieses Textes, bei der es sich um eine unbeglaubigte Abschrift handelt. Diese teilt sich mit der zweiten Ausfertigung die Signatur A 602 Nr. 461a.

37 Vgl. Anm. 5. 
Konzept als auch die Ausfertigung bei der Übergabe des Herzogtums von Eberhard auf Ulrich diesen als Herzog an, hatte der König ihm doch bereits am 28. Mai das württembergische Herzogtum verliehen; das zweite Konzept bezeichnete Ulrich zunächst ebenfalls als Herzog, dieser Titel wird aber dann mittels Streichung durch Graf ersetzt. Diese Veränderung innerhalb des zweiten Konzepts findet sich dort durchgehend an allen Stellen, an denen von Ulrich die Rede ist. Offenbar sollte so die Fiktion eines freiwilligen, erst durch den Horber Vertrag sich vollziehenden Übergangs des Herzogtums von Eberhard an Ulrich untermauert werden. Besonders Eberhard musste daran ein Interesse haben, weswegen dieses Spezifikum des zweiten Konzepts auf ihn zurückgehen dürfte; dagegen hat sich die Gegenseite jedoch erfolgreich gewehrt, wie der ausgefertigte Text zeigt.

Einzig das erste Konzept erwähnt auch, dass Ulrich bis zu seinem 20. Lebensjahr ein Regiment an die Seite gestellt werden solle. Dieser Passus wurde indes weder in das zweite Konzept noch in die Ausfertigung übernommen, vermutlich weil sowohl Ulrich als auch der König darin eine zu große Beschränkung ihrer künftigen Handlungsfreiheit sahen, und weil außerdem ein entsprechender, freilich weniger präzise formulierter Hinweis bereits Aufnahme in die Belehnungsurkunde vom 28. Mai 1498 gefunden hatte ${ }^{38}$.

Nicht im ersten, wohl aber im zweiten Konzept ist im Anschluss an diese Stelle eine sehr weitreichende Bestimmung zugunsten Eberhards enthalten. Demnach sollte das Erbrecht eines ehelichen Sohnes, sofern Eberhard noch einen solchen zeugen sollte, von der Übertragung der Herrschaft auf Ulrich unberührt bleiben; einem solchen Sohn und dessen männlichen Nachkommen hätte damit die Herrschaft über Württemberg eingeräumt werden müssen. Zwar wurde diese Regelung auch in die Ausfertigung übernommen, freilich mit einer wesentlichen Einschränkung, die darauf hinauslief, dass sie keine Wirksamkeit entfalten konnte; in der Urkunde vom 10. Juni 1498 heißt es nämlich, dass diese Bestimmung nur für Söhne aus der aktuellen Ehe Eberhards mit der Markgräfin Elisabeth von Brandenburg gültig sein sollte. Angesichts der Kinderlosigkeit der bis dato 31 Jahre währenden Ehe, des zerrütten Verhältnisses der beiden Eheleute ${ }^{39}$, des Umstands, dass Elisabeth ihrem Gemahl nicht ins Exil gefolgt war, und nicht zuletzt im Hinblick auf das Alter der 1451 geborenen Hohenzollerin war die Möglichkeit der Geburt eines solchen Sohnes faktisch ausgeschlossen. Hingegen wäre es 1498 durchaus denkbar

${ }^{38}$ Darin heißt es, dass der König Wolfgang von Fürstenberg und das Regiment als Verwalter der Herrschaft über Württemberg eingesetzt habe und zwar so lang Er und seine zugeordneten Rete das vorberürt Regiment des Furstenthumbs Wirttemberg auff unsern obbestimpten beuelch ond Vertrag verwalten oder bis der gemelt Graue Ulrich zu seinen vogtpern Jaren kommet; SAtTler (wie Anm. 3) Beyl. 14. Tatsächlich erklärte Maximilian Ulrich dann bereits mit 16 Jahren für mündig und die Regentschaft für beendet. Vgl. dazu sein Schreiben an die Regenten vom 16. Juni 1503: HStA Stuttgart G 41 Bü 1 Bl. 4.

39 So vermochten es Eberhards Räte 1496 nur mit Mühe, die von ihm beabsichtigte Trennung von Elisabeth zu verhindern. Vgl. dazu: HStA Stuttgart A 602 Nr. 445. 
gewesen, dass nach einem etwaigen frühzeitigen Tod Elisabeths ${ }^{40}$ Eberhard in einer weiteren Ehe noch Söhne geboren worden wären, was zeigt, wie sehr gerade das zweite Konzept Eberhards Interessen entgegenkam.

Aufschlussreich mit Blick auf den Verhandlungsprozess sind auch die darauf folgenden Angaben zu den Pensionszahlungen, die Eberhard gemäß dem Vertrag für seinen Regierungsverzicht zustehen sollten. Im ersten Konzept ist noch von 4.000 rheinischen Gulden jährlich die Rede, die er jeweils in vier gleichen Raten erhalten sollte. Im zweiten Konzept lautete die Angabe zunächst 8.000 Gulden, also das Doppelte, wurde dann aber gestrichen und mit einem Platzhalter (.N.) versehen. Die Ausfertigung nennt 6.000 Gulden. Hier lassen sich die Verhandlungsabläufe gut nachvollziehen: Die 4.000 Gulden des ersten Konzepts waren Eberhard ganz offensichtlich zu wenig, so dass es ihm zunächst gelang, eine Verdoppelung des Betrages im zweiten Konzept durchzusetzen, das dadurch einmal mehr als ein Text erscheint, der die Interessen Eberhards weit stärker berücksichtigte als das erste Konzept. Diese Forderung dürfte jedoch bei Ulrich und seinem Regiment auf Ablehnung gestoßen sein, weswegen man die Summe strich und die Entscheidung darüber dem König anheimstellte. Dafür spricht u.a., dass sich Eberhard am 16. Juni 1498 über die seiner Ansicht nach zu niedrig angesetzte Summe von 6.000 Gulden beschwerte ${ }^{41}$, was ihm kaum möglich gewesen wäre, wenn er selbst dieser Pensionshöhe als Kompromiss zugestimmt hätte. In jedem Fall war das Ergebnis dann eine Summe, die exakt die Mitte zwischen den beiden Beträgen des ersten und des zweiten Konzepts darstellte.

Besser durchsetzen konnte sich Eberhard bei der freilich weniger bedeutsamen Frage des Übergabeortes der Pensionszahlungen. Zunächst war mit Ulm eine dem württembergischen Territorium nahe gelegene Reichsstadt vorgesehen. Im zweiten Konzept sollte die Übergabe dagegen zunächst im Umkreis eines Ortes nach Eberhards Wahl erfolgen. Bei einer Überarbeitung des zweiten Konzepts wurde dies dahingehend geändert, dass nun Eberhard aus drei - noch mit Platzhaltern versehenen Städten - den Übergabeort bestimmen können sollte. Schließlich legte die ausgefertigte Urkunde Eberhards künftigen hauptsächlichen Wohnsitz als Übergabeort fest. Auch hier berücksichtigten das zweite Konzept und die Ausfertigung Eberhards Interessen stärker als das erste Konzept.

Der nächste Punkt im ersten Konzept, die Bestimmung, dass aller Besitz, über den Eberhard bei seinem Tod verfügen würde, nach Abzug der Schulden an Ulrich fallen solle, ist im zweiten Konzept und der Ausfertigung weiter nach hinten gerückt. Ansonsten hat sich aber an der Regelung nicht Wesentliches geändert, so dass vermutet werden darf, dass diese zwischen den Parteien nicht strittig war.

40 Tatsächlich starb Elisabeth erst 1524 und damit 20 Jahre nach Eberhard, so dass die Regelung des zweiten Konzepts auch keine andere Wirkung entfaltet hätte als die der Ausfertigung, was 1498 indes nicht vorhersehbar war.

${ }^{41}$ HStA Stuttgart A 602 Nr. 464. 
Anders verhält es sich mit der im ersten Konzept nun folgenden Verpflichtung Ulrichs und seines Regiments, für Eberhards Schulden aufzukommen, die im zweiten Konzept und der Ausfertigung gleichfalls erst an späterer Stelle im Text auftaucht. Inhaltlich war zunächst vorgesehen, dass die neuen württembergischen Herren nicht für diejenigen Schulden Eberhards haften sollten, die dieser vor seinem Regierungsantritt verursacht, ferner auch nicht für diejenigen, so er inn mittler zytt für sich selbs gemacht hatte, sowie schließlich für diejenigen Verbindlichkeiten, die er nach dem Verlassen des Landes eingegangen war bzw. eingehen würde. Auch hier trat im zweiten Konzept insofern eine Änderung zugunsten Eberhards ein, die dann auch in die Ausfertigung übernommen wurde, als die Nennung der während seiner Regierungszeit entstandenen Privatschulden unter den von Eberhard zu tragenden Lasten entfiel. Dies war auch insofern sinnvoll, als eine entsprechende Bestimmung mit großer Sicherheit ein beständiger Quell künftiger Streitigkeiten zwischen den Parteien über die Frage gewesen wäre, welche Forderungen als Privatschulden anzusehen sein würden. Schon im Hinblick darauf dürfte die Streichung geboten erschienen sein.

Gleichfalls im zweiten Konzept und in der Ausfertigung textlich weiter nach hinten gerückt sind die im ersten Konzept nun folgenden Bestimmungen über den Umgang mit dem von Eberhard aus dem Lande geführten Silbergeschirr und anderen Kleinodien. Darüber hinaus haben sich hier inhaltliche Veränderungen ergeben: Sprach das erste Konzept noch davon, dass diese Gegenstände Eberhard zunächst als Nießbrauchsgut zur Verfügung gestellt werden und nach seinem Tod - oder einer schweren Vertragsverletzung durch Eberhard - Ulrich und dem Herzogtum wieder zukommen sollten, so war im zweiten Konzept zunächst davon die Rede, dass ein Inventar dieser Gegenstände erstellt und Ulrich übergeben werden sollte. Auch diese Bestimmung wurde indes bereits im zweiten Konzept überarbeitet und diese Modifikation dann in die Ausfertigung übernommen, wonach die Gegenstände nun Maximilian zuzustellen waren, damit er eine Teilung vornehmen könne.

Demgegenüber sind die Veränderungen in den Bestimmungen zur Versorgung von Eberhards Gemahlin Elisabeth und der seines Bruders Heinrich rein redaktioneller Natur. So wird vor allem bei Elisabeth ab dem zweiten Konzept ihr Fürstenstand besonders betont, dem die Versorgung angemessen sein müsse. Insgesamt dürfte hier indes Einigkeit zwischen den Parteien bestanden haben, vermutlich nicht zuletzt deshalb, weil mit dieser Vereinbarung keine neuen Lasten verbunden waren.

In die Ausfertigung wurde im Anschluss daran die Regelung aufgenommen, dass Eberhard auf Lebenszeit Württemberg nicht wieder betreten dürfe. Diese eindeutig als Sicherung der neuen württembergischen Herren gedachte Bestimmung dürfte auf diese zurückgehen. Bereits im ersten Konzept war ein solches Verbot vorgesehen. Eine Ergänzung des ersten Konzepts ging sogar noch weiter, indem sie dieses Verbot auf das ganze lannd zu Swauben ausdehnte, also auf ein Gebiet, das 
deutlich über das württembergische Territorium hinausreichte. Im zweiten Konzept findet sich hierzu keine Parallele, was erneut unterstreicht, dass dieses stärker die Interessen Eberhards berücksichtigte als das erste und als die Ausfertigung.

Deutlich wird diese Tendenz auch bei den im Text nun folgenden Bestimmungen zu den Personen, die während des vorangegangenen Konflikts auf Eberhards Seite gestanden hatten und nun in württembergischen Gefängnissen saßen. Hier nannte das erste Konzept eine Reihe von Anhängern Eberhards (Andreas Nagel, ferner einen Forstmeister von Urach ${ }^{42}$, Hans Trompter, Bertelin Engelfried sowie einen Siechengrett), die alle in den beiden anderen Texten nicht mehr namentlich auftauchen. Für sie wurde im ersten Konzept ihre Freilassung vorgesehen, allerdings sollten sie wie auch andere, nicht genannte Gefangene Schwaben dauerhaft verlassen. Im zweiten Konzept wird nicht nur keiner von ihnen mehr mit Namen genannt, vielmehr werden auch die Regelungen für den Umgang mit den gefangenen Anhängern Eberhards flexibler gehandhabt, indem deren Schicksal allein in die Hand des Königs gelegt wurde; dieser sollte nun entscheiden, was zu veranlassen sei, damit sie dem neuen Regiment nicht mehr gefährlich werden könnten. Die Ausfertigung stellte das Schicksal dieser Personen gleichfalls dem König anheim; er hatte freilich über die Bestimmungen des zweiten Konzepts hinaus Sorge dafür zu tragen, dass diese Personen auf Lebenszeit nicht mehr nach Württemberg kommen könnten. Auch hier findet sich also eine Verschärfung in der Ausfertigung gegenüber dem zweiten Konzept zugunsten der neuen württembergischen Herren, weswegen damit zu rechnen ist, dass diese auch auf sie zurückgeht.

Besonders deutlich zeigt sich die eberhardfreundliche Tendenz des zweiten Konzepts schließlich bei den Bestimmungen zu zwei weiteren Anhängern, die im ersten Konzept und in der Ausfertigung namentlich genannt und dort auch mit spezifischen Sanktionen versehen werden, während sie im zweiten Konzept in der anonymen Masse der von Ulrich und seinem Regiment Gefangengehaltenen verschwinden und somit für sie dort das gleiche gilt wie für die anderen Anhänger Eberhards. Hierbei handelt es sich um Konrad Holtzinger, einen Augustinermönch und engen Vertrauten Eberhards, sowie um Hans von Stetten. Der zuletzt genannte etwa sollte dem ersten Entwurf gemäß sein Leben lang im Dorf Stetten bleiben sowie Ulrich und das Regiment mit einer ihrer Sicherheit dienenden Verschreibung ausstatten, während der Text für Holtzinger die Bitte Maximilians an den Bischof von Konstanz vorsah, ihn inn öwiger gefängknuß zu halten oder an Ulrich auszuliefern, der ihn gleichfalls lebenslänglich gefangenhalten sollte. Dagegen findet in der Ausfertigung die Ordenszugehörigkeit Holtzingers größere Berücksichtigung, indem nun bestimmt wird, dass er von seinem Orden lebenslänglich vencklichen gehallten werden solle. Etwas günstiger sind die Bestimmungen der Ausfertigung

42 Dabei dürfte es sich um Melchior Lendorfer handeln, von dem auch eine Urfehde aus dieser Zeit erhalten ist (HStA Stuttgart A 602 Nr. 4288). Vgl. dazu auch: Neues Württembergisches Dienerbuch, bearb. von Walther Pfeilsticker, 3 Bde., Stuttgart 1957/1963/1993, $\$ 2958$. 
für Hans von Stetten: Er sollte eine Urfehde schwören, dann aber freigelassen werden, mit der Auflage, Württemberg nie mehr zu verlassen ${ }^{43}$.

Änderungen im Verlauf des Verhandlungsprozesses gab es schließlich auch bei den Bestimmungen zu jenen Anhängern Eberhards, die im Zusammenhang mit den vorangegangenen Streitigkeiten das Land verlassen und durch das neue Regiment Vermögensverluste erlitten hatten. Diesen sollte ihr Vermögen prinzipiell restituiert werden. Bei den Voraussetzungen hierfür und dem Umfang der Restitution ergeben sich freilich Unterschiede zwischen dem ersten Konzept einerseits und dem zweiten Konzept sowie der Ausfertigung andererseits: Gemäß dem ersten Konzept sollten diese Personen noch eigens eine Verschreibung ausstellen, nie mehr gegen die Interessen Württembergs zu handeln und auch dann nur jenes Vermögen zurückerhalten, so noch vorhanden ist. Dagegen war nach dem zweiten Konzept bzw. der Ausfertigung der allgemein übliche Huldigungseid gegenüber Ulrich und dem Regiment ausreichend; eine Einschränkung bei den zu restituierenden Gütern gab es nun nicht mehr. Allerdings wurde zunächst auch im zweiten Konzept die Abgabe einer Urfehdeerklärung der betreffenden Personen gefordert, diese Passage dann allerdings bei einer Überarbeitung ersatzlos gestrichen. Erneut zeigt sich hier das erste Konzept somit deutlich stärker von den Wünschen Ulrichs und des Regiments geprägt als das zweite Konzept und die Ausfertigung, auch wenn hier die Abmilderung teilweise erst bei den Redaktionsarbeiten zum zweiten Konzept erfolgte.

In der nächsten Passage, die den Umgang mit den Scheltworten regelt, die beide Parteien im Laufe des vorangegangenen Konflikts übereinander geäußert hatten, gibt es keine größeren Abweichungen zwischen den drei Textversionen. Dies dürfte seinen Grund darin haben, dass eine entsprechende Regelung, wonach der Vermittler die vorausgegangenen Beleidigungen der Ehre keiner der beiden Seiten abträglich zu sein erklärte, zum gängigen Inhalt eines Schiedsvertrags in dieser Zeit gehörte $^{44}$. Das gleiche gilt für die sich anschließende Feststellung Maximilians, dass durch die Vereinbarung beide Seiten wieder miteinander vertragen und die Irrungen geschlichtet seien.

Der folgende Abschnitt des ersten Konzepts (und zugleich sein letzter erhaltener) widmet sich dem Problem der Verpflichtung der Untertanen auf Eberhard bzw. untereinander im Bündnis gegen ihn. Dieses Bündnis gegen ihn sollte ebenso

43 Die entsprechende Urfehde Stettens vom 28. Juni 1498 ist abgedruckt bei: SATTLER (wie Anm. 3) Beyl. 17. Weitere erhaltene Urfehden, die mit dem geschilderten Konflikt in Zusammenhang stehen, sind diejenigen von Melchior Lendorfer (vgl. Anm. 42) sowie Andreas Nagel (HStA Stuttgart A 602 Nr. 4289), beide vom 8. August 1498.

${ }_{44}$ Zur Bedeutung der fürstlichen Ehre gerade im ausgehenden Mittelalter und den bisweilen bestehenden Schwierigkeiten der Vermittler, hier zu befriedigenden Übereinkünften zu kommen, vgl. Jean Marie Moeglin, Fürstliche Ehre und verletzte Ehre der Fürsten im spätmittelalterlichen Deutschen Reich, in: Verletzte Ehre. Ehrkonflikte in Gesellschaften des Mittelalters und der Frühen Neuzeit, hg. von Klaus Schreiner/Gerd Schwerhoff (Norm und Struktur, Bd. 5), Köln u.a. 1995, S. 77-91. 
aufgehoben sein wie die Pflichten der Untertanen gegenüber Eberhard. Eine entsprechende Passage findet sich so weder im zweiten Konzept noch in der Ausfertigung. So wurde die Aufhebung der Verpflichtung seiner Untertanen auf Eberhard in den beiden späteren Texten bereits im Zusammenhang mit der ersten Bestimmung, dem Rücktritt Eberhards von der Regierung, geregelt. Der Hinweis auf eine Conjuratio der Untertanen gegen Eberhard entfiel im zweiten Konzept und der Ausfertigung sogar gänzlich, vermutlich deshalb, weil er letztlich keinem Beteiligten als günstig für die eigene Interpretation des Herrschaftsübergangs erschienen sein dürfte.

Die folgenden Passagen finden sich aufgrund des Fragmentcharakters des ersten Konzepts nur noch im zweiten Konzept und in der Ausfertigung. Dabei handelt es sich zum einen um die Festlegung, dass beide Parteien Maximilian die Kompetenz zum Entscheid von etwaigen Streitfragen in der Auslegung des Urkundentextes überlassen, zum anderen um die Strafandrohung im Falle des Bruchs der Vereinbarung durch eine der Parteien. In diesen relativ festen Bestandteilen einer Schiedsurkunde ergeben sich keine inhaltlichen Änderungen zwischen dem zweiten Konzept und der Ausfertigung. Die Abweichungen in der die Urkunde beschließenden Datierung erklären sich problemlos aus den sachlichen Veränderungen des Ausstellungsortes und -datums ${ }^{45}$; auch der im Konzept gegenüber der Ausfertigung fehlende Hinweis auf die Regierungsjahre des Königs verwundert nicht sonderlich, da starre formelhafte Bestandteile in Entwürfen häufig weggelassen wurden. Für die Interpretation wesentliche inhaltliche Unterschiede im Eschatokoll sind zwischen dem zweiten Entwurf und der Ausfertigung freilich nicht erkennbar.

\section{Resümee}

Der Vergleich der drei vorliegenden Texte lässt verschiedene Rückschlüsse auf die Genese des Horber Vertrags zwischen Ende Mai und dem Ausstellungsdatum des 10. Juni 1498 zu. Demnach wurde in der Kanzlei Maximilians zunächst ein erstes Konzept entworfen, in das offenbar schon die Interessen Ulrichs bzw. seines Regiments einflossen, deren Vertreter spätestens seit der Ankunft Maximilians in Ulm Anfang Mai den König ständig begleiteten und mit ihm bzw. den königlichen Räten am Hof Verhandlungen pflegten. Dementsprechend berücksichtigte dieses Konzept die Interessen Eberhards vergleichsweise wenig. Im Rahmen einer ersten Überarbeitung, deren Stand die Streichungen und Ergänzungen im ersten Konzept repräsentieren, wurden dann sogar einige Bestimmungen weiter zulasten Eberhards verschärft, was vermutlich auch auf den Einfluss der neuen württember-

45 Eine gewisse Beachtung könnte hier allenfalls der Umstand finden, dass das Konzept nach Heiligen-/Festtagen datiert, während die Ausfertigung die moderne Form der Datierung nach Tag und Monat wählt. 
gischen Herren zurückzuführen ist. Dagegen trägt das zweite Konzept den Interessen Eberhards in weit größerem Umfang Rechnung. Es ist daher davon auszugehen, dass er erst jetzt intensiver in den Verhandlungsprozess einbezogen wurde und seinen Forderungen - durchaus mit gewissem Erfolg - Geltung zu schaffen suchte, wenn es ihm auch nicht gelang, alle ihm wichtigen Punkte durchzusetzen. Vielmehr war es vor der Ausfertigung der Urkunde offenbar den neuen württembergischen Herren möglich, noch einmal Stellung zu nehmen und einige der zugunsten Eberhards lautenden Bestimmungen wieder zu tilgen oder in ihrem Sinne abzumildern ${ }^{46}$. Dieser Umstand wiederum sowie die fehlende Berücksichtigung anderer Forderungen Eberhards im Horber Vertrag bildeten die Grundlage für sein Beschwerdeschreiben an den König vom 16. Juni, mit dem er freilich keine Änderung mehr herbeizuführen vermochte, zumal er ja bereits in Kenntnis des Horber Vertrages am 11. Juni seinen Rücktritt erklärt hatte.

Der Horber Vertrag selbst präsentiert sich somit an verschiedenen Stellen als ein Kompromiss zwischen dem ersten und dem zweiten Konzept - besonders gut ablesbar an der Höhe der jährlichen Pensionszahlungen für Eberhard -, der vermutlich auf den vermittelnden Einfluss des Königs bzw. seiner Umgebung zurückzuführen ist. Insgesamt gesehen änderte dies jedoch nichts daran, dass mit dem Horber Vertrag die de facto-Absetzung eines Reichsfürsten besiegelt wurde, die nicht nur ihm ungerecht erschien, sondern die - wie die Äußerungen Schurlins von Leonberg und Herzog Ulrichs zu Eingang dieses Beitrags verdeutlichen - auch von anderen politischen Akteuren als aufsehenerregendes und keineswegs über jeden moralischen Zweifel erhabenes Ereignis wahrgenommen wurde.

\section{Anhang: Textsynopse}

Die folgende Synopse gibt die Texte in einer gemäßigt normalisierten Form wieder. Sie orientiert sich dabei im Wesentlichen an der aktuellen Transkriptionsrichtlinie der Archivschule Marburg ${ }^{47}$. Auf die Wiedergabe späterer Registratur- und Archivvermerke wurde verzichtet. Im Falle von Korrekturen und Änderungen an den Texten wird der jeweils letzte Stand in der Synopse geboten, während die früheren Varianten im Apparat zu finden sind.

${ }^{46}$ Darüber hinaus bot sich hier noch einmal die Möglichkeit, kleinere sachliche Unstimmigkeiten zu beseitigen, wie eine Korrektur im Zusammenhang mit den linksrheinischen Besitzungen des Hauses Württemberg verdeutlicht. War bis zum zweiten Entwurf noch von den Grafschaften Mömpelgart und Reichenweier (im Plural) die Rede, so wurde dem Umstand, dass tatsächlich nur Mömpelgart den Rang einer Grafschaft besaß, in der Korrektur des zweiten Entwurfs Rechnung getragen und anschließend nurmehr der Singular verwendet.

47 „Grundsätze für die Textbearbeitung im Fach Historische Hilfswissenschaften“, Pkt. II, veröffentlicht unter: http://www.archivschule.de/uploads/Ausbildung/Grundsaetze_ fuer_die_Textbearbeitung_2009.pdf (Stand: 8.12.2013). 


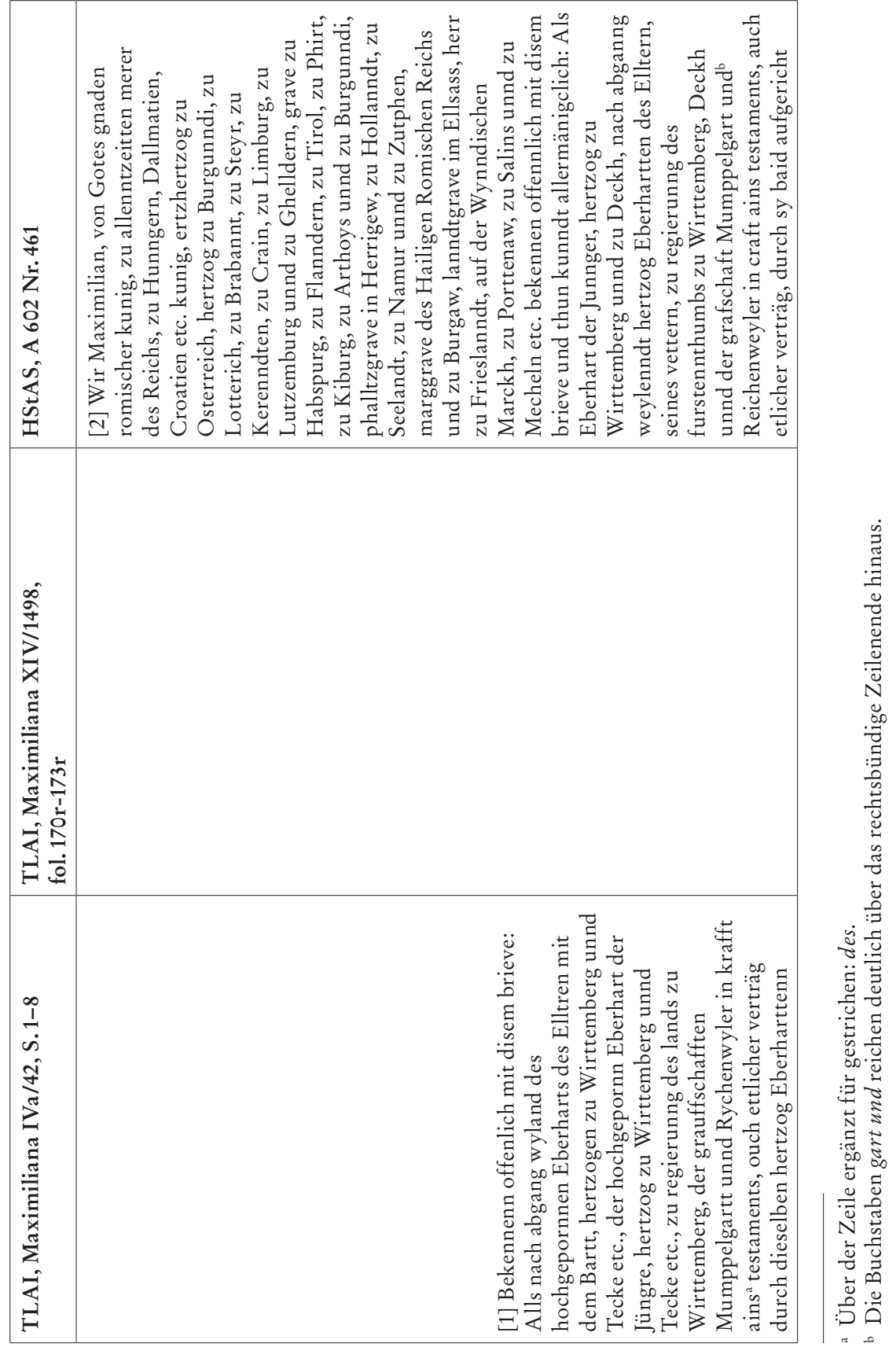




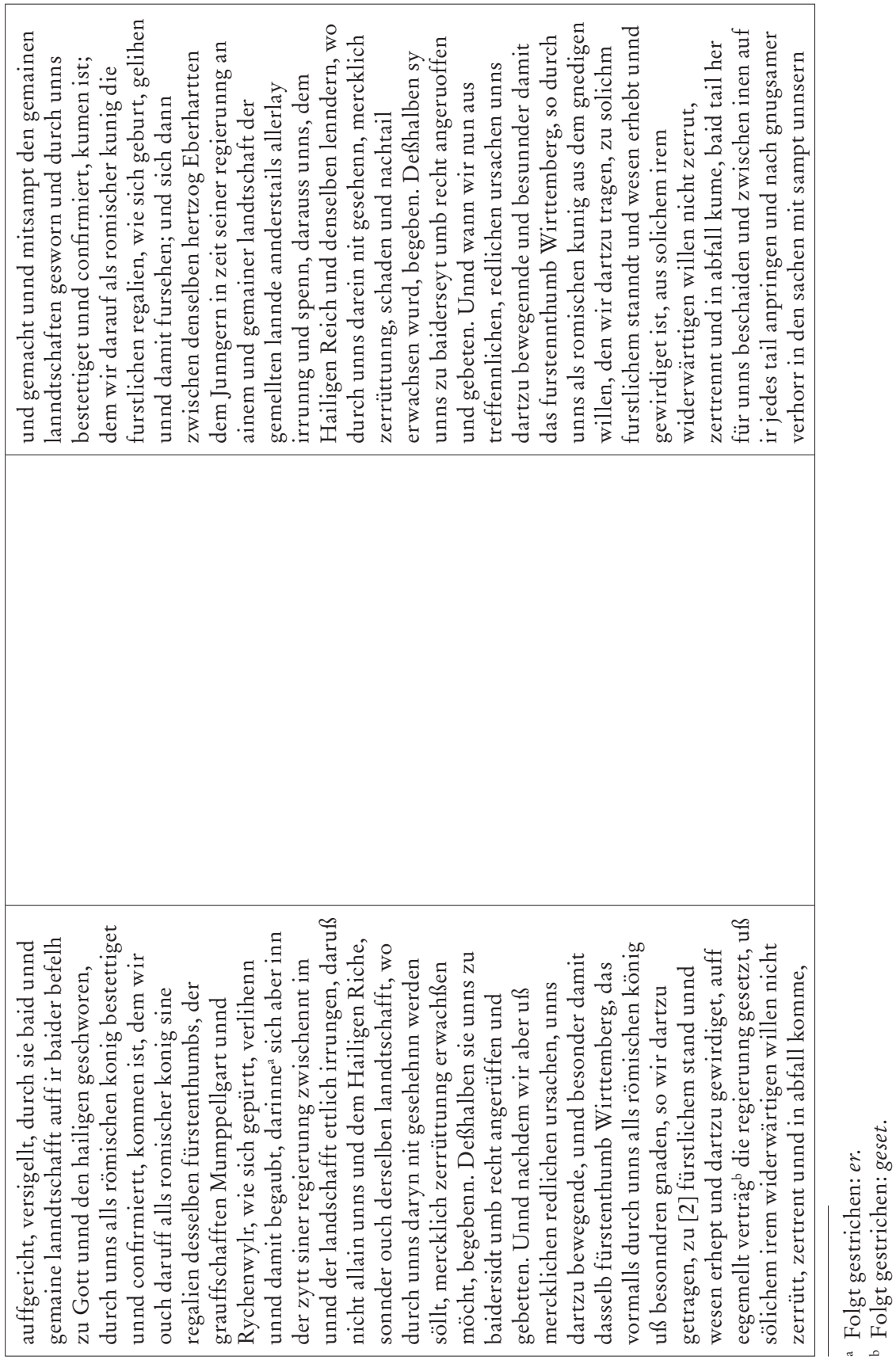




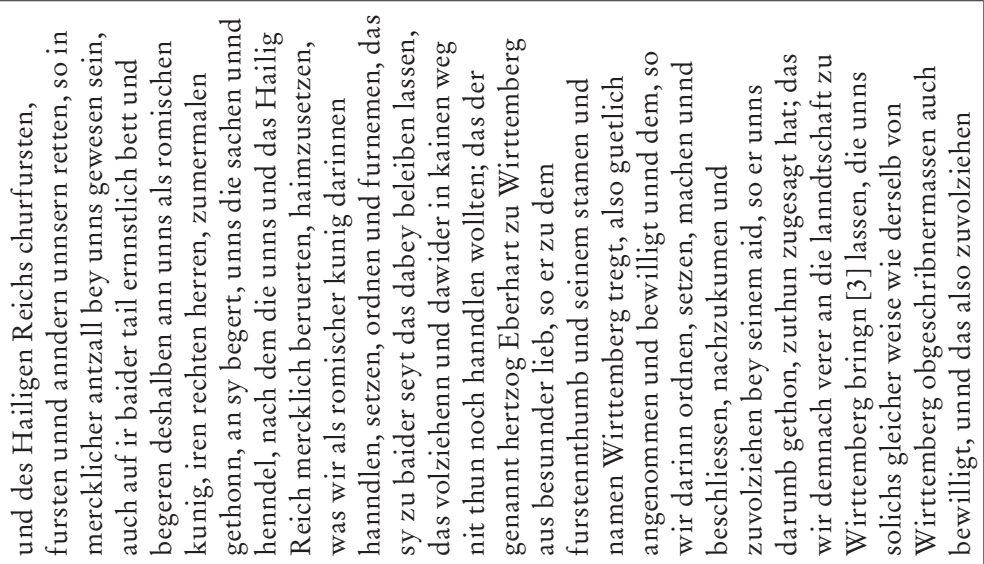

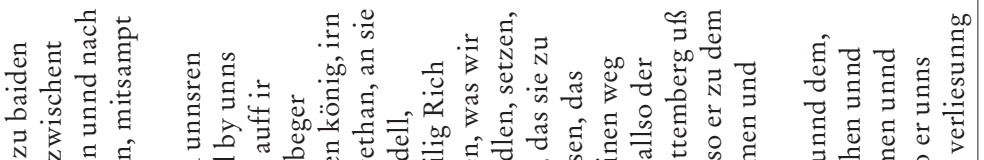

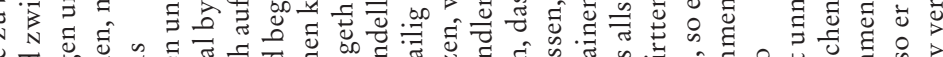
.

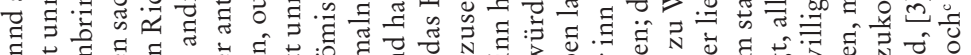

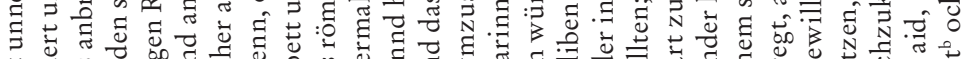

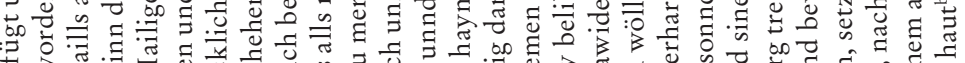

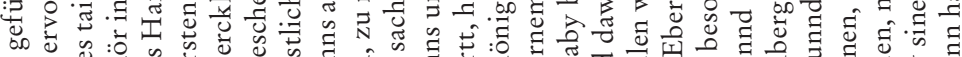

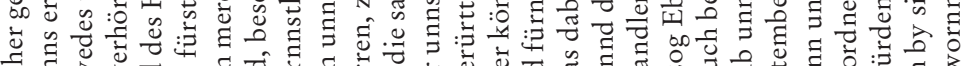

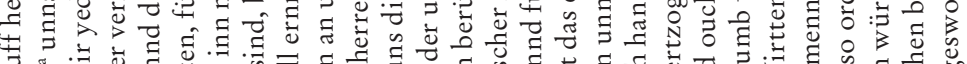

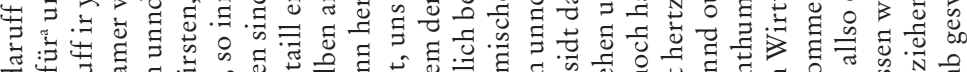

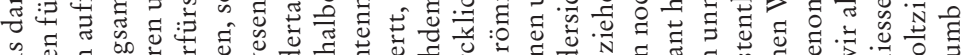

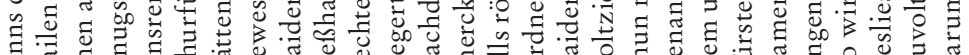



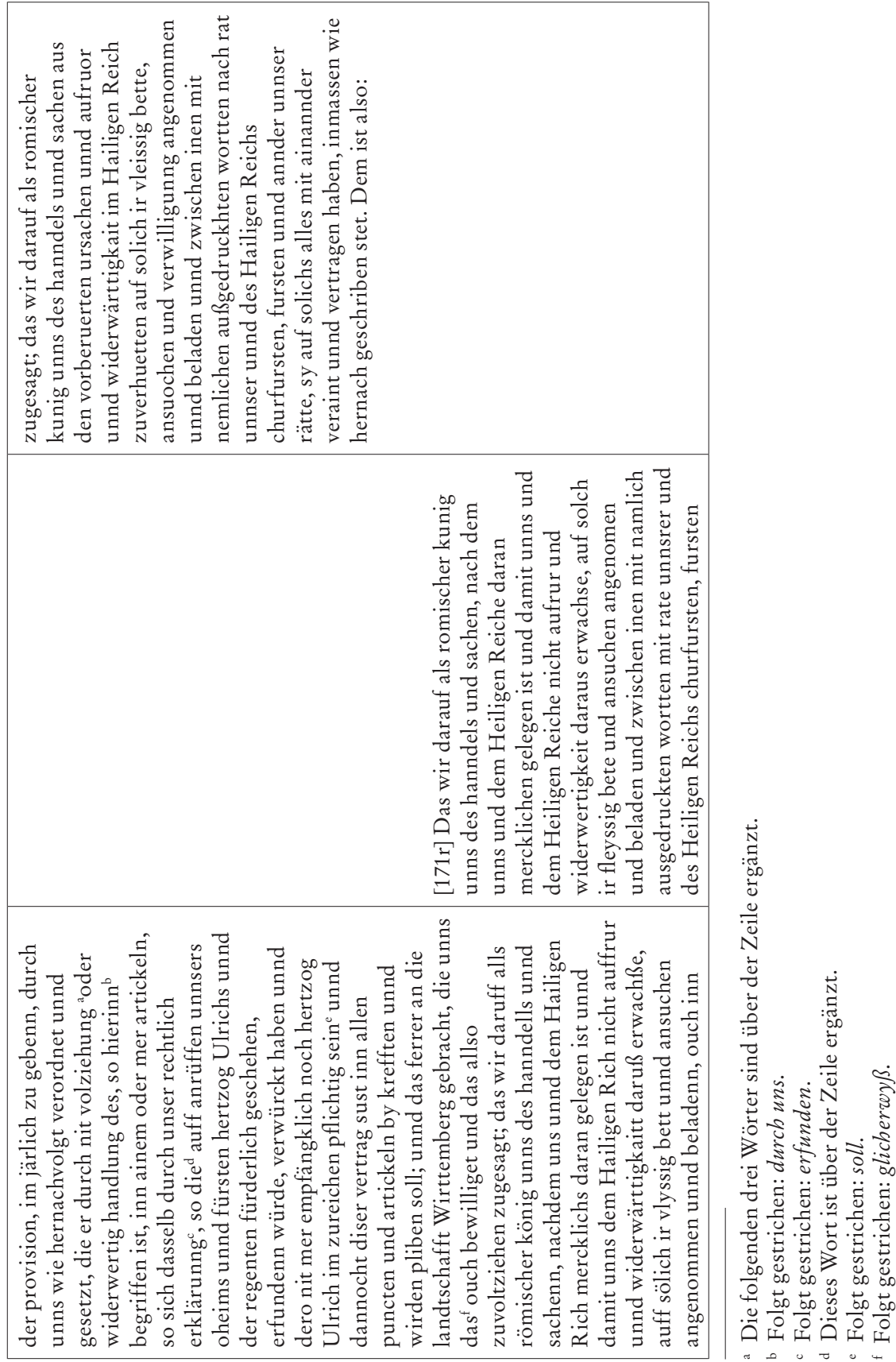


\begin{tabular}{|c|c|}
\hline & 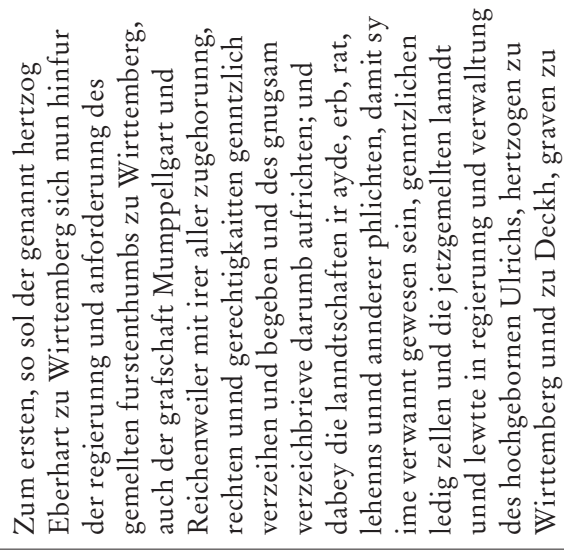 \\
\hline 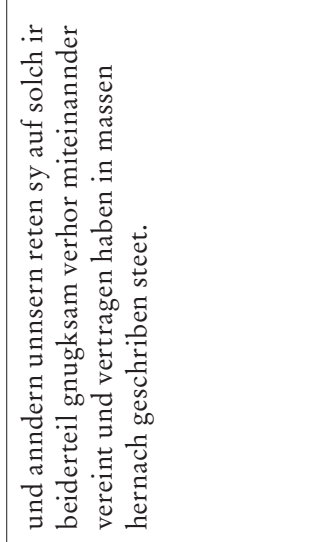 & 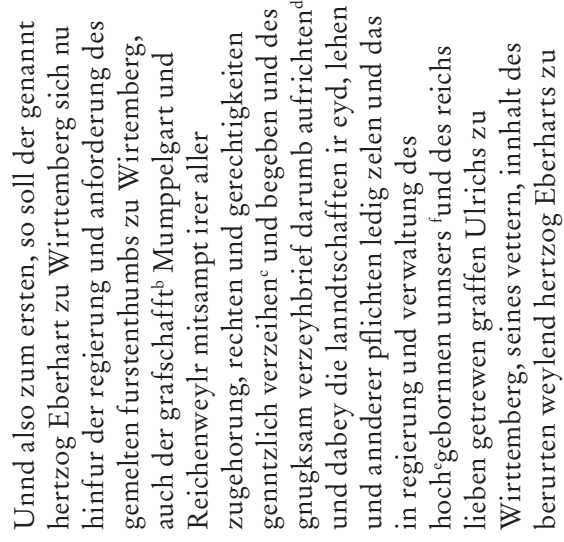 \\
\hline 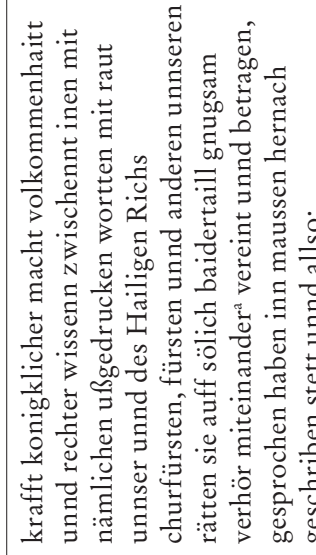 & 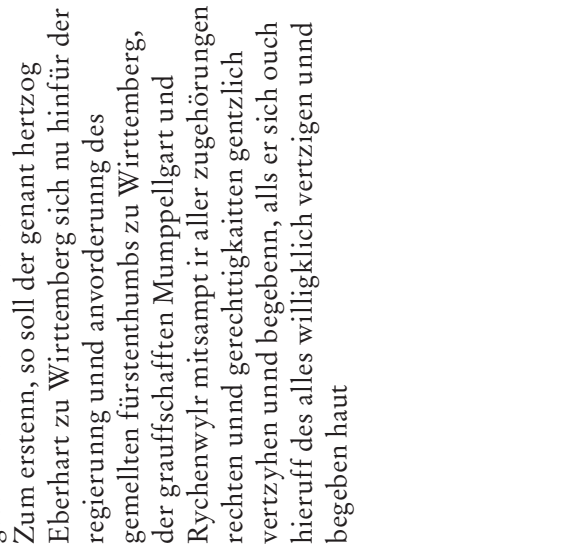 \\
\hline
\end{tabular}

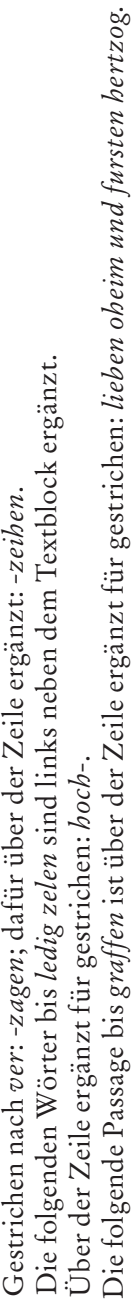




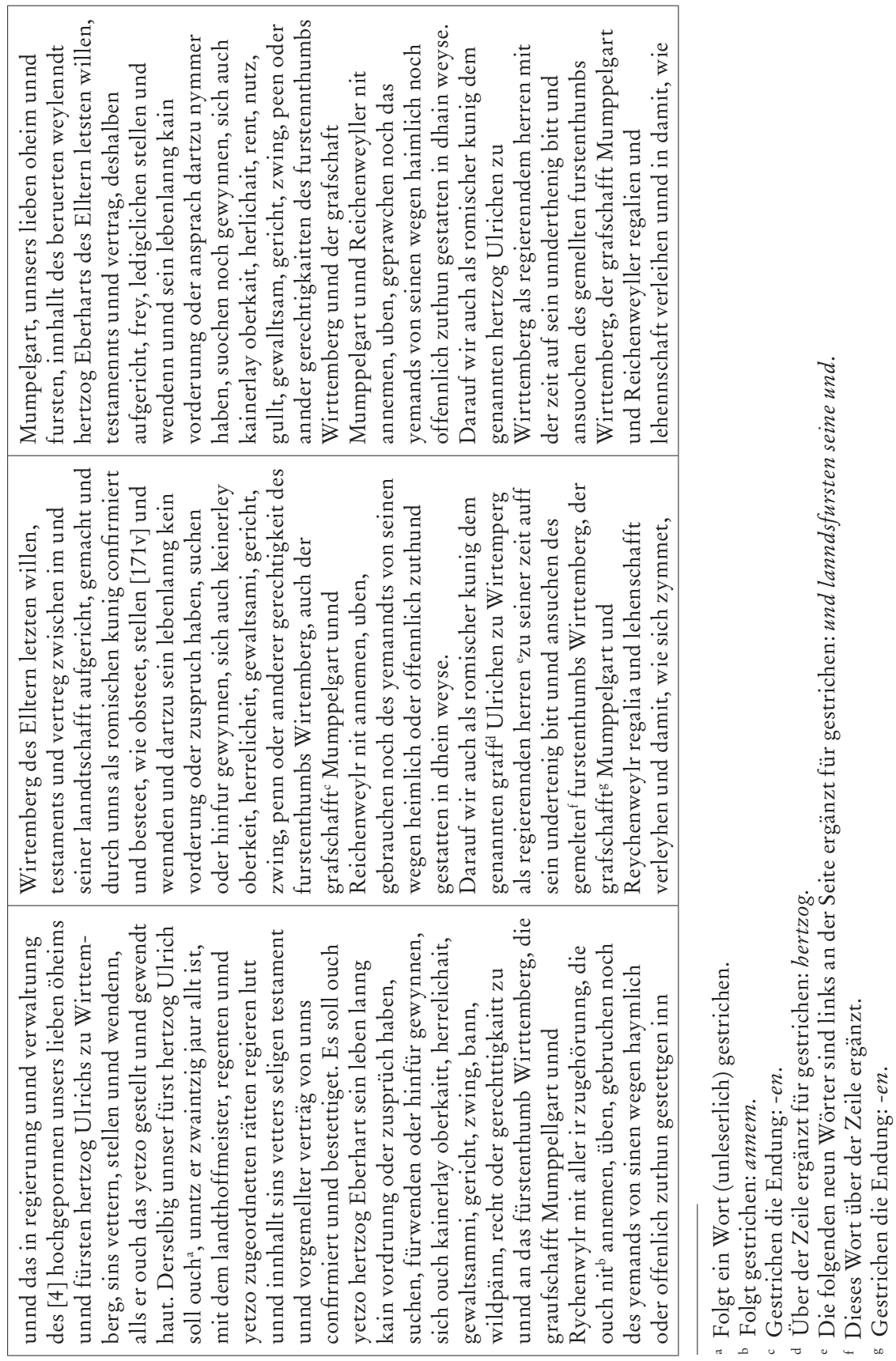




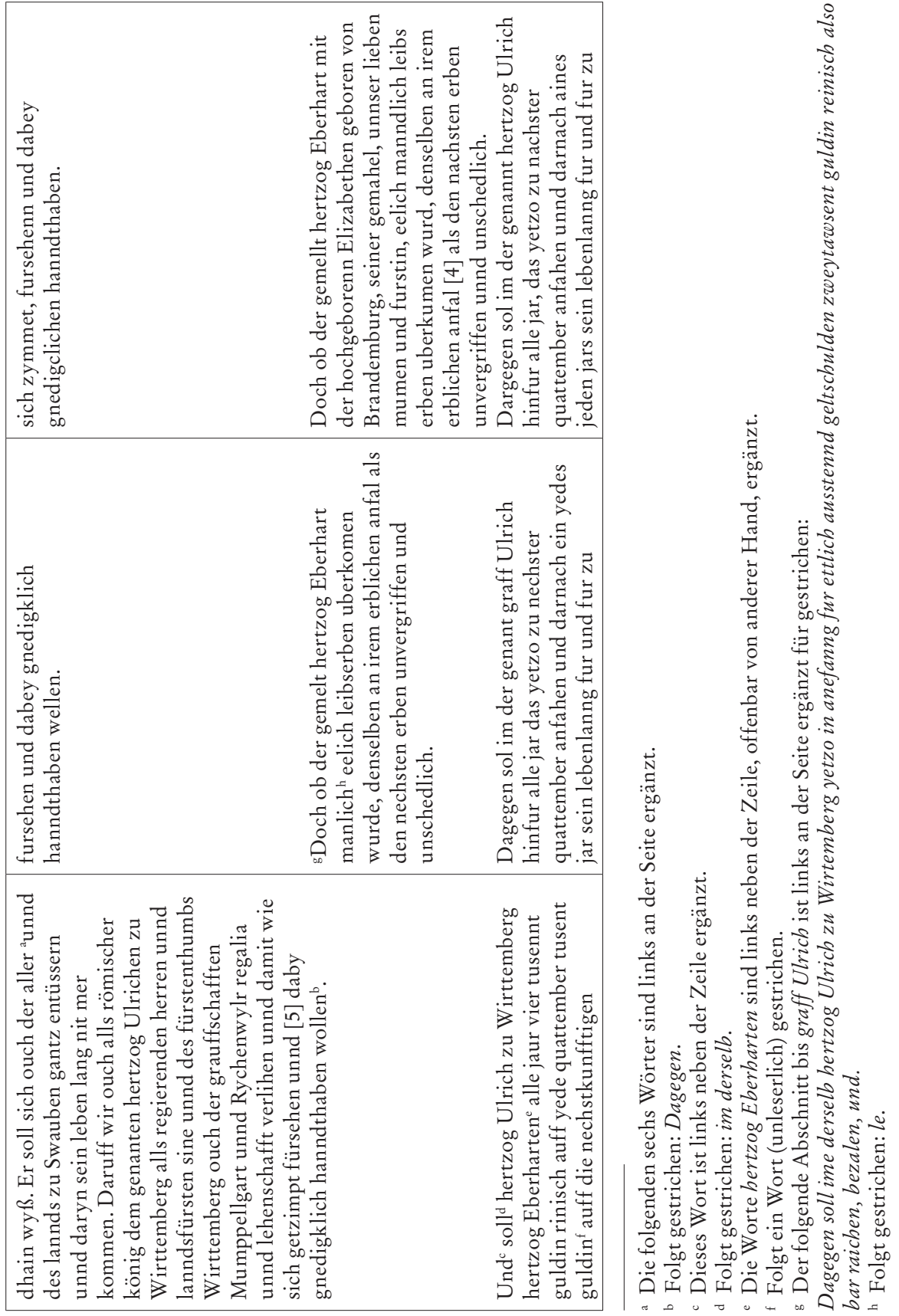




\begin{tabular}{|c|c|c|}
\hline \multicolumn{2}{|c|}{ 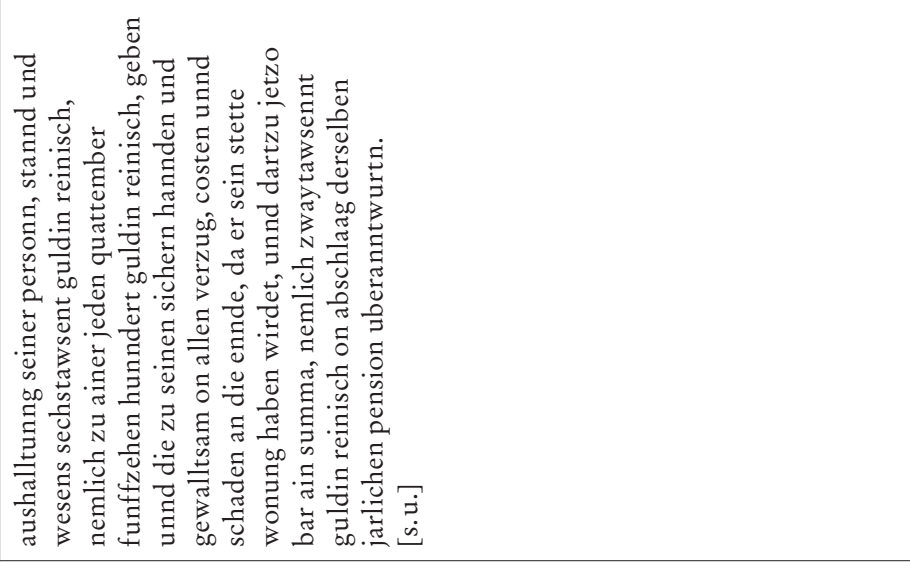 } & 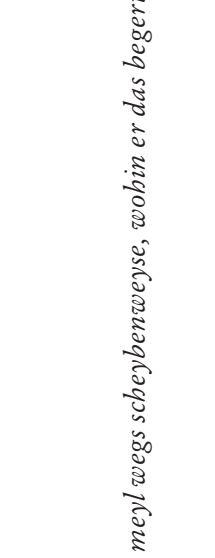 \\
\hline 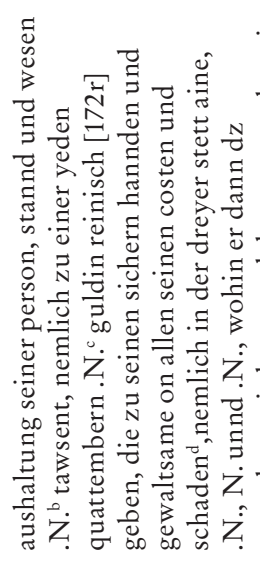 & $\begin{array}{l}\stackrel{\dot{j}}{\dot{\omega}} \\
\dot{\dot{\omega}}\end{array}$ & 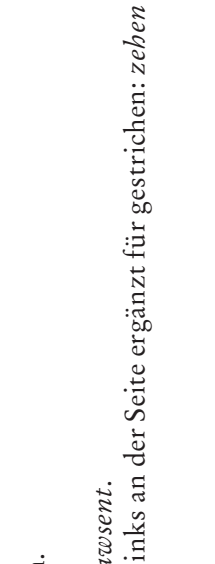 \\
\hline 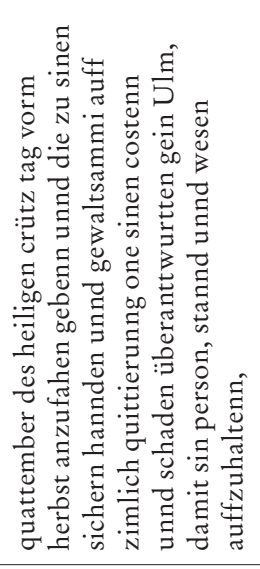 & 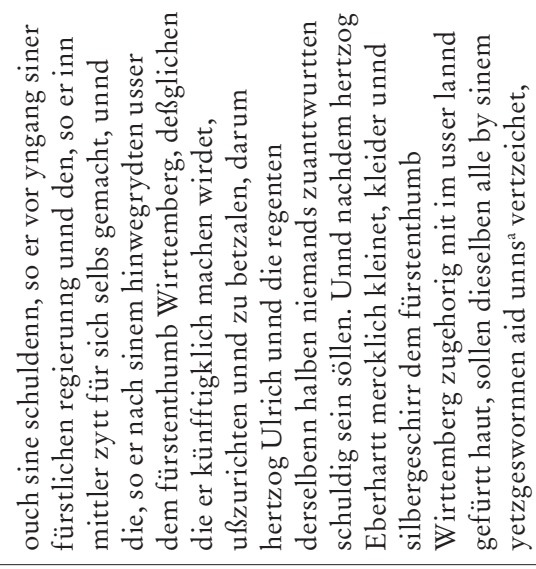 & 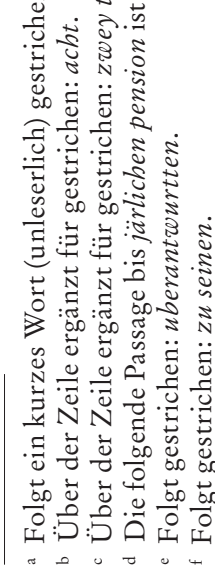 \\
\hline
\end{tabular}




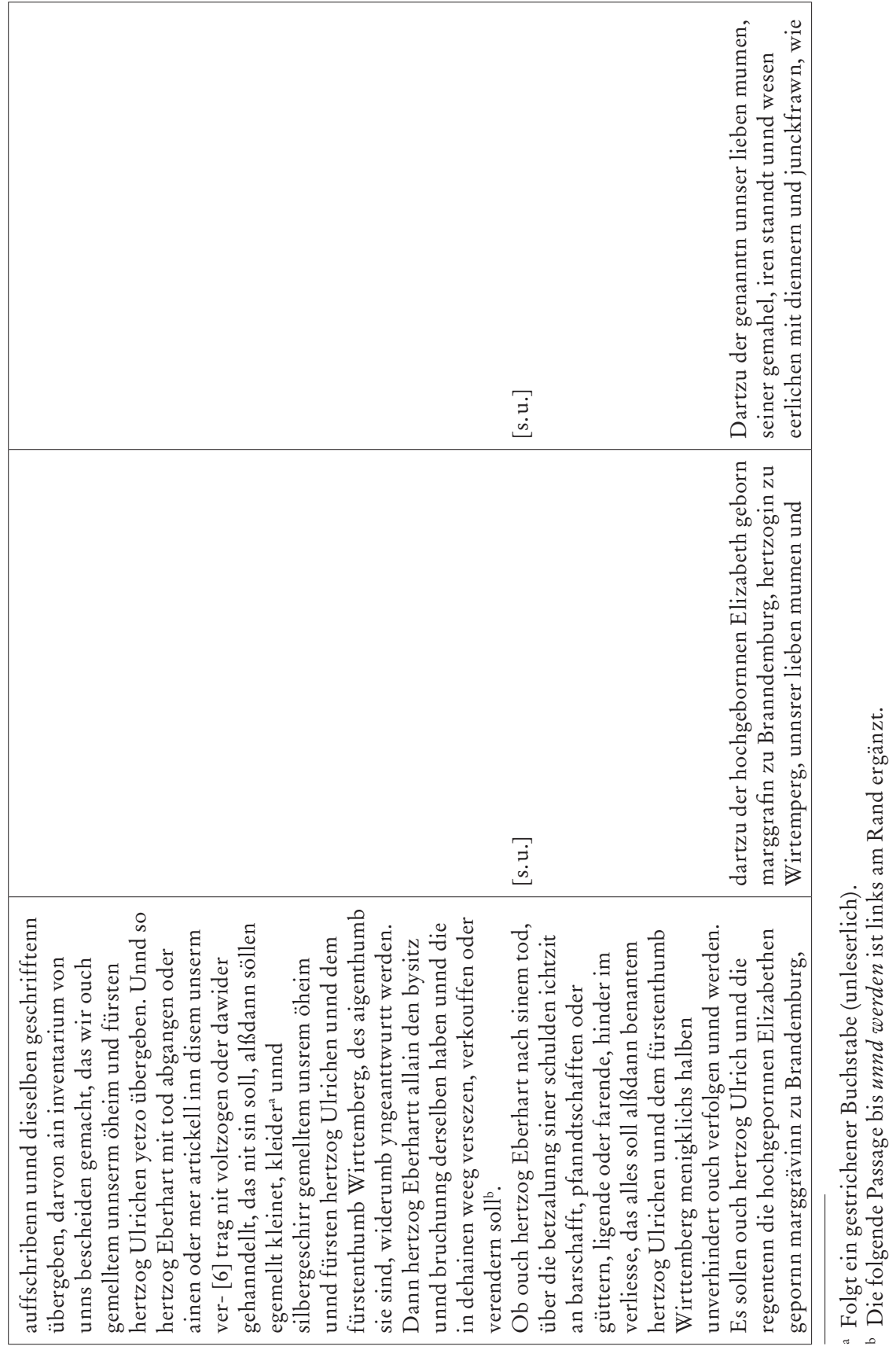




\begin{tabular}{|c|c|}
\hline 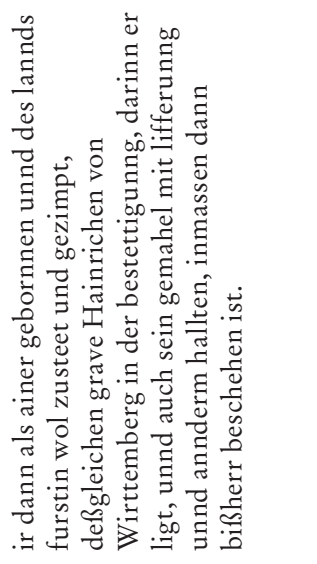 & 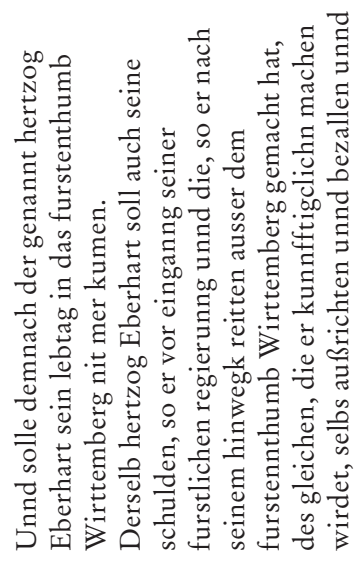 \\
\hline 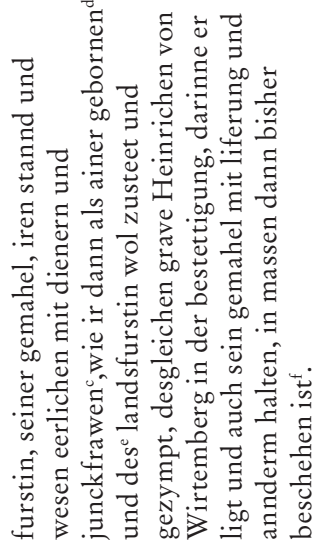 & 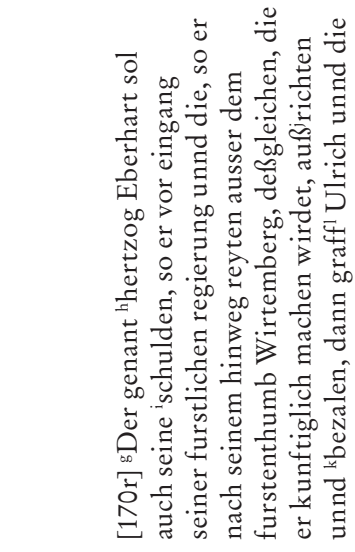 \\
\hline 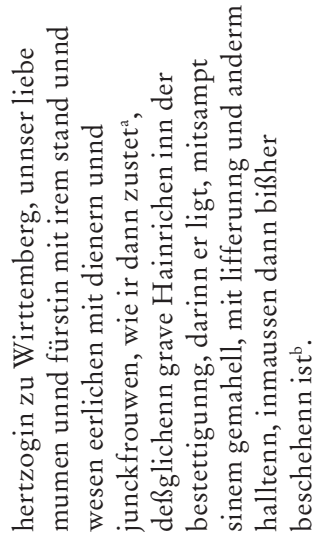 & $\begin{array}{l}\dot{\varphi} \\
\dot{\varphi}\end{array}$ \\
\hline
\end{tabular}

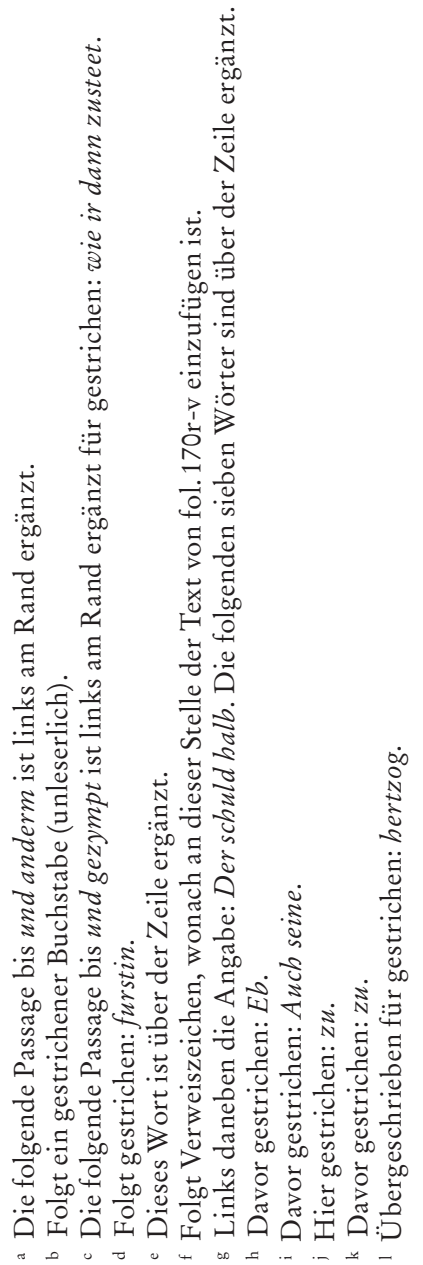




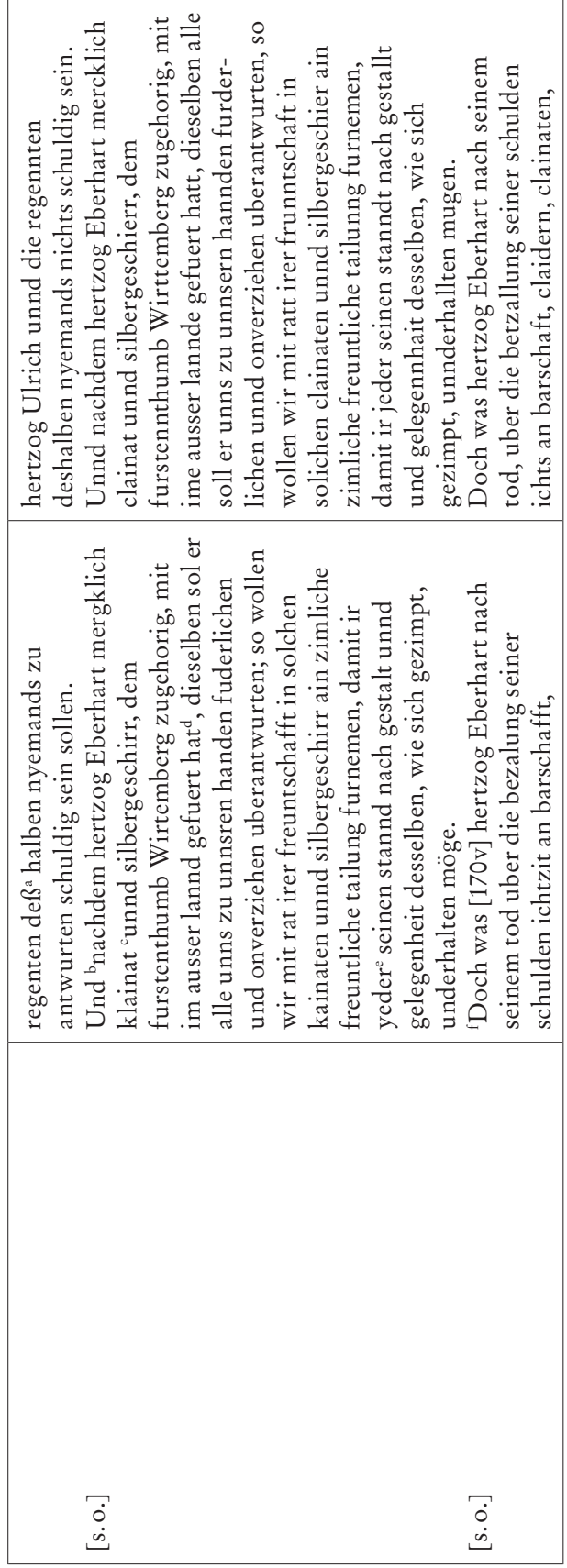

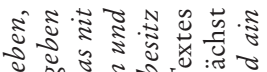
०.

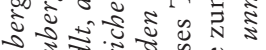
3. 농

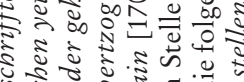

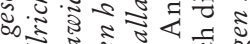

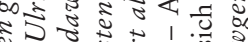
-

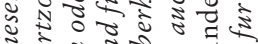

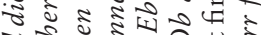
ช 啇 N

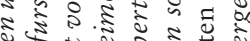

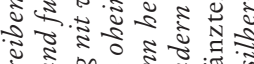

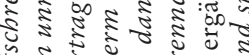

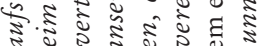

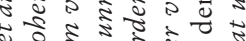

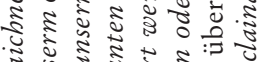
और है है है उ

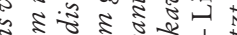
ปี है

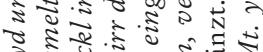
วิ

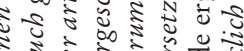

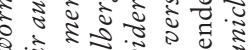

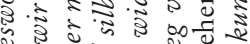

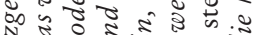

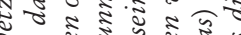
กิ

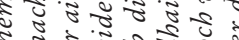

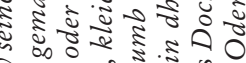

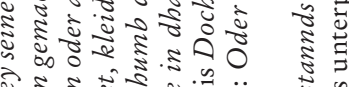
इं

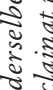

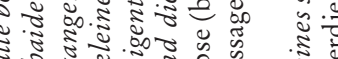

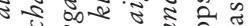
काष

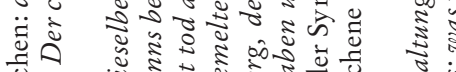

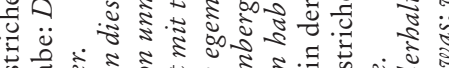

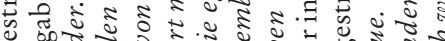

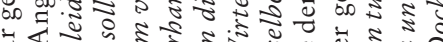

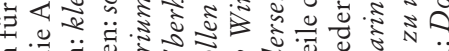

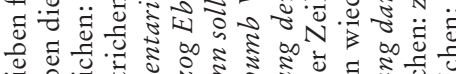

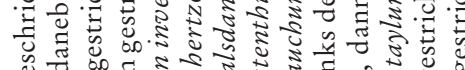

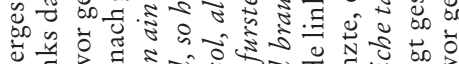

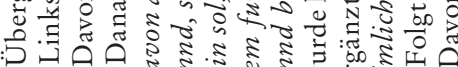




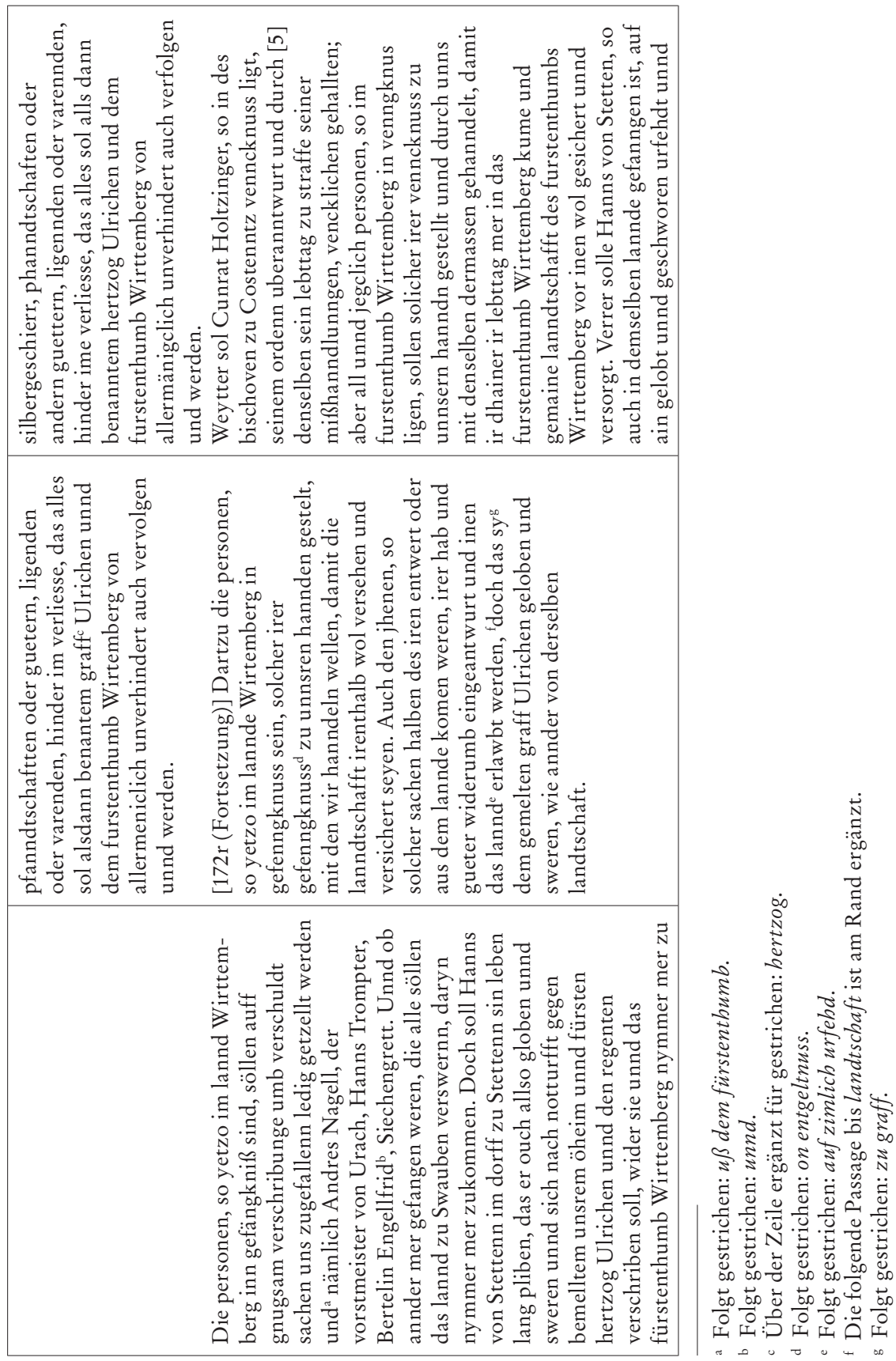




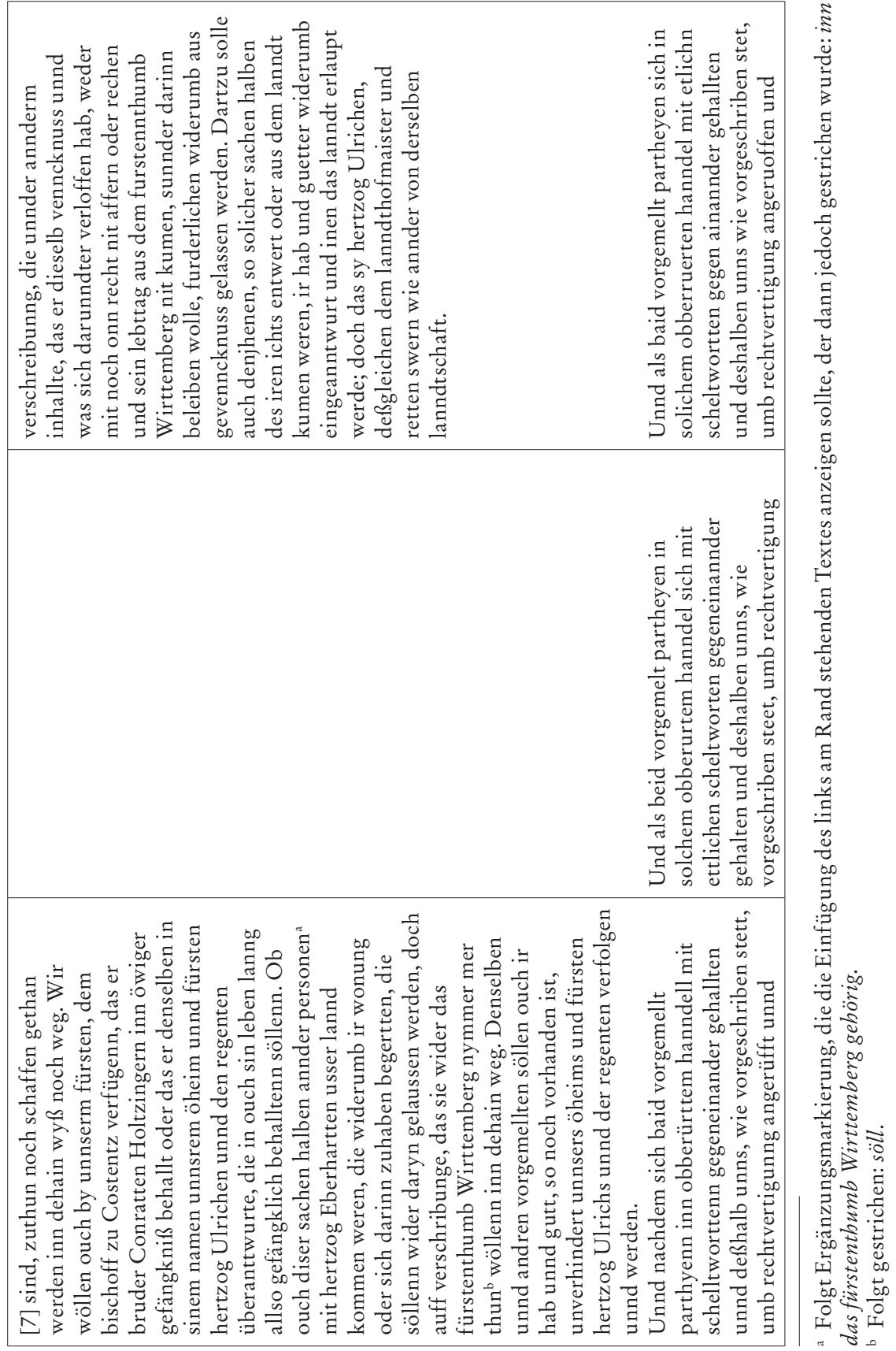




\begin{tabular}{|c|c|c|}
\hline 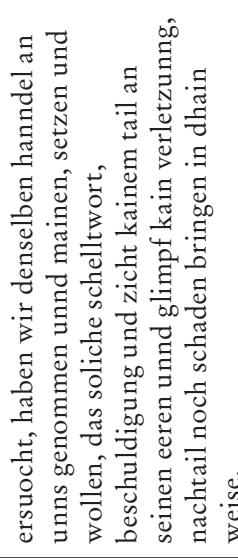 & 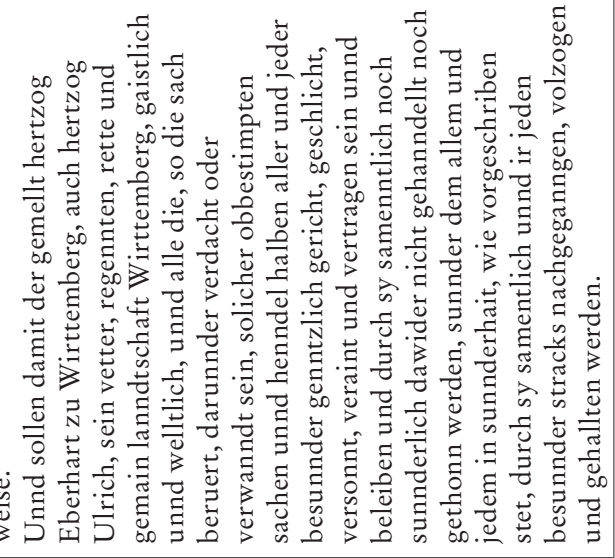 & \\
\hline 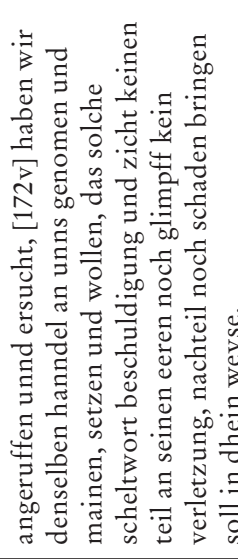 & 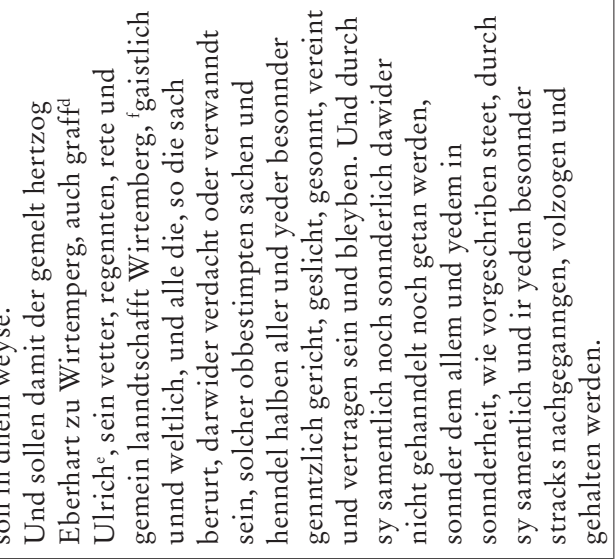 & 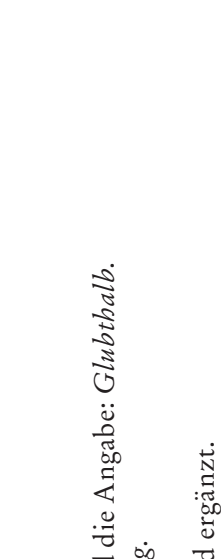 \\
\hline 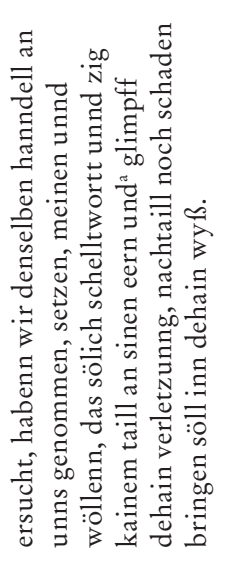 & 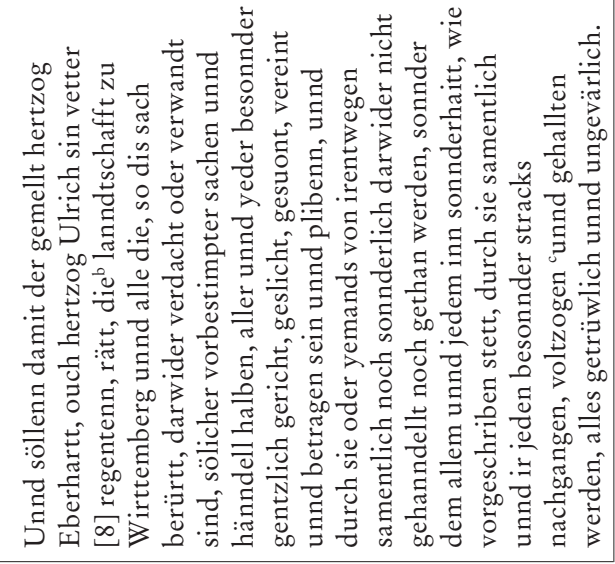 & 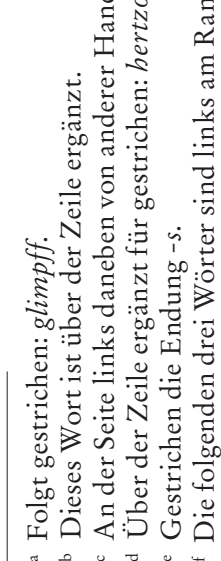 \\
\hline
\end{tabular}



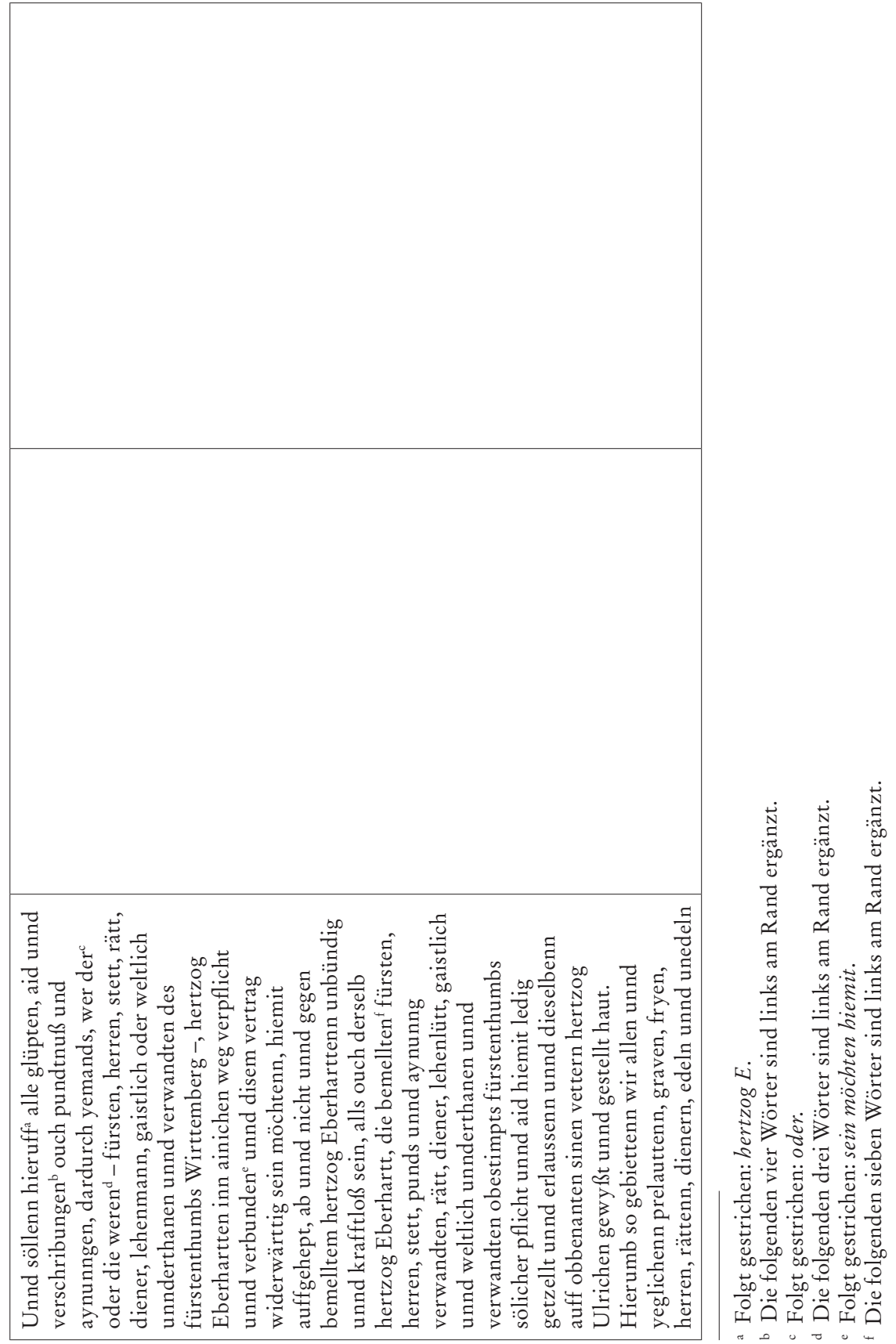


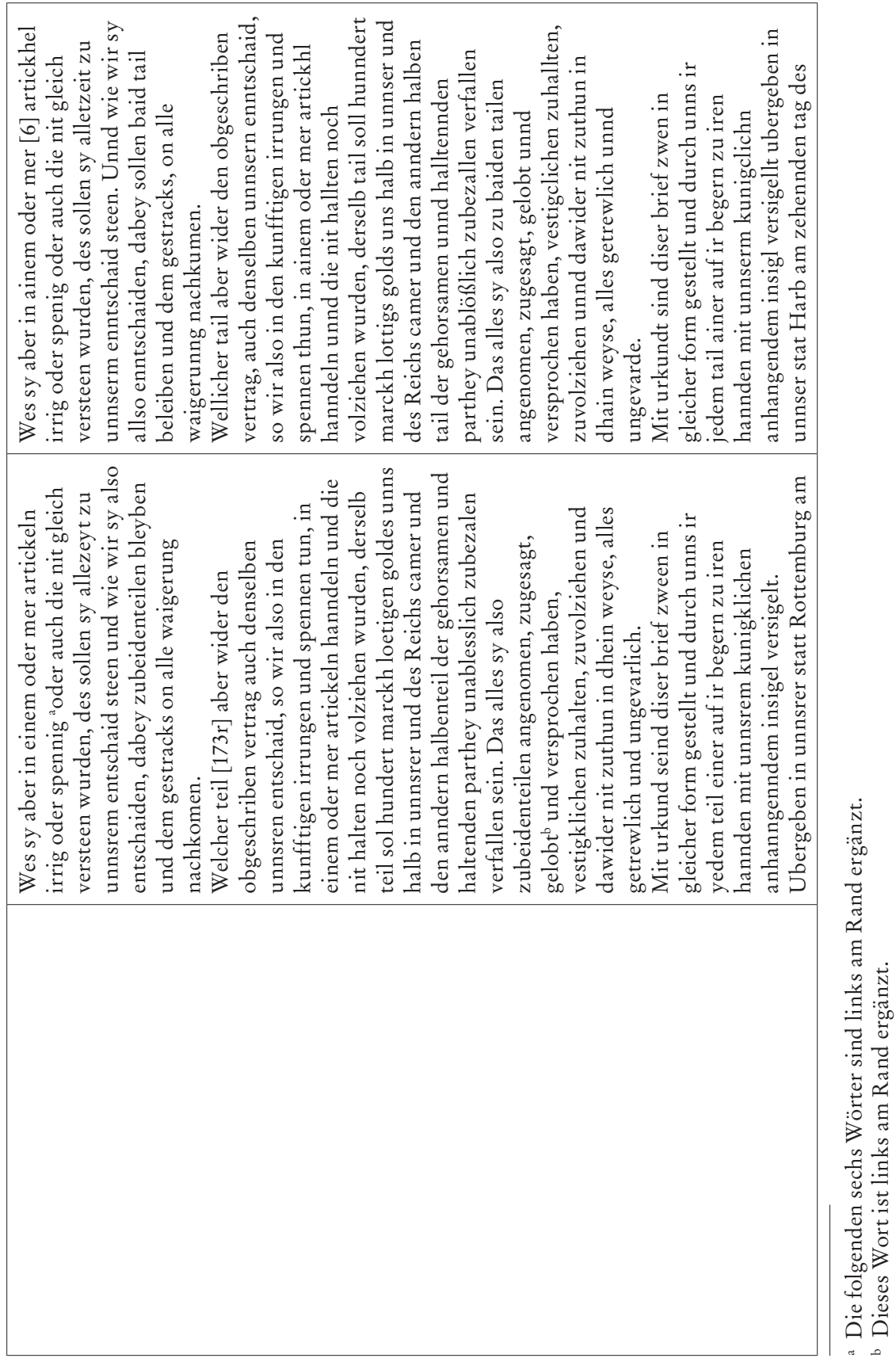




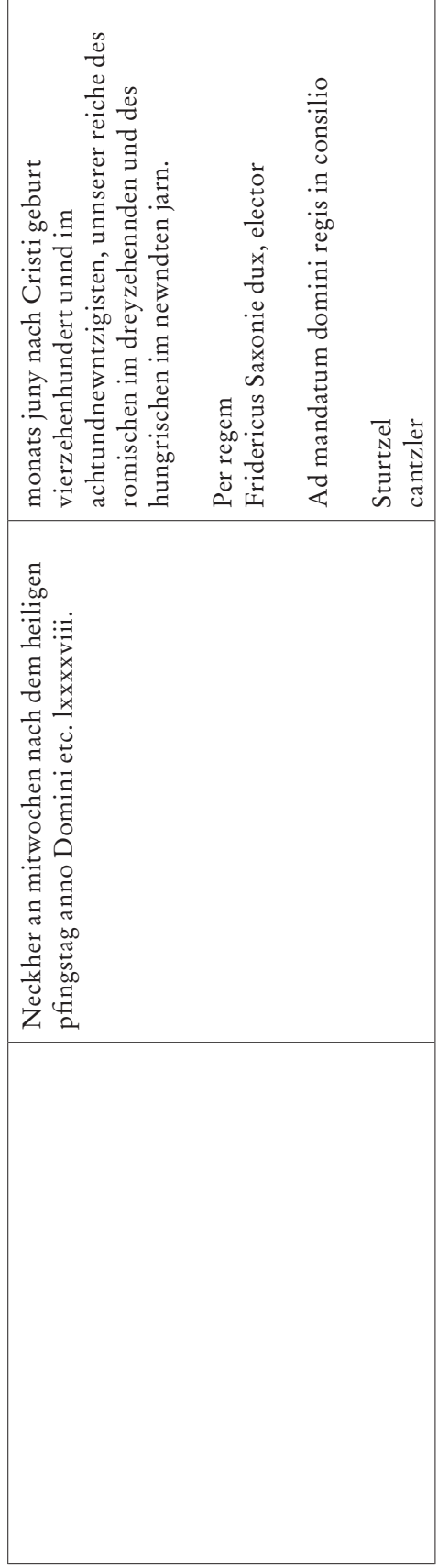

